\title{
Active Set Solver for Min-Max Robust Control with State and Input Constraints
}

\author{
Johannes Buerger $^{1}$, Mark Cannon ${ }^{2, *}$, Basil Kouvaritakis ${ }^{2}$ \\ ${ }^{1}$ BMW Group, Munich, Germany \\ ${ }^{2}$ Department of Engineering Science, University of Oxford, OX1 3PJ, UK
}

\begin{abstract}
SUMMARY
This paper proposes an online active set strategy for computing the dynamic programming solution to a min-max robust optimal control problem with quadratic $\mathcal{H}_{\infty}$ stage cost for linear systems with linear state and input constraints in the presence of bounded disturbances. The solver determines the optimal active constraint set for a given plant state using an iterative procedure which computes the optimal sequence of feedback laws for a candidate active set and updates the active set by performing a line search in state space. The computational complexity of each iteration depends linearly on the length of the prediction horizon. The main contribution of the paper is its treatment of degeneracy caused by linearly dependent state and input constraints and its efficient handling is a crucial step in formulating the active set algorithm. The proposed approach ensures the continuity of optimal control laws along the line-of-search, thus enabling an efficient solution method based on homotopy. Conditions for global optimality are given and the convergence of the active set solver is established using the geometric properties of an associated multi-parametric programming problem. A receding horizon control strategy is proposed, which ensures a specified $l_{2}$-gain from the disturbance input to the state and control inputs in the presence of linearly dependent constraints. Copyright (c) 0000 John Wiley \& Sons, Ltd.
\end{abstract}

Received...

KEY WORDS: robust control, min-max optimization, dynamic programming, receding horizon control

\section{INTRODUCTION}

The aim of robust control based on min-max optimality is to minimize the worst-case performance of the controlled system over a known set of unknown future disturbances [27, 18, 2] while satisfying constraints robustly. Due to its significant practical importance, this area has been a major challenge to researchers for decades and the system-theoretical side of this problem is by now well-understood [22], while its computational aspects still pose considerable challenges. The major challenge lies in the fact that the optimal solutions to min-max control problems have the form of feedback laws [18] and, using available dynamic programming methods, the computation needed to optimize over arbitrary feedback laws typically grows exponentially with the system state dimension and horizon length.

For the important class of linear systems with additive, bounded uncertainty there remains the challenge of obtaining computationally efficient solutions which ideally solve the underlying dynamic programming problem exactly or at least approximate it to a high degree of accuracy. In the scenario-based approach of [24], an exact solution is obtained which involves impracticably large computational loads since the numbers of decision variables and constraints grow exponentially

*Corresponding author: mark. cannon@eng.ox.ac.uk 
with the horizon length. Therefore a variety of suboptimal formulations have been proposed as computationally tractable min-max control strategies. The contributions of $[17,21,13,23]$ select dynamic programming policy parametrizations and optimize over the free parameters. Most nobably in the context of robust MPC these parametrizations consist of: the class of pre-stabilizing policies [21]; policies with linear state feedback parameters [17]; the more general disturbance-affine policies of [19, 13]; and the more general separable disturbance feedback parametrization of [23].

As an alternative to solving min-max robust control problems online via dynamic programming, approaches based on multiparametric programming have been proposed $[1,9]$. These use offline computations to characterize the optimal control law, typically as a piecewise affine state feedback law. However this requires the optimal control law to be determined for all feasible system states, and moreover it relies on the ability to efficiently determine which of many polyhedral regions contains the current state. Although efficient point location techniques have been proposed (e.g. [9, 14]), the method is generally applicable only to small problems and short horizons.

The current paper shows that optimal solutions can be determined efficiently by exploiting the linearity and continuity properties of dynamic programming solutions using an active set method based on homotopy. This avoids the disadvantages of suboptimal problem formulations as well as those of offline dynamic programming approaches that rely on multiparametric programming. Moreover we demonstrate that the computation required by each iteration of the proposed algorithm grows only linearly with the horizon length of the control problem.

Methods based on homotopy of solutions for nominal (i.e. uncertainty-free) control problems [8, $10,3]$ were extended to the case of linear systems with bounded additive uncertainty and linear input constraints in [7], and to linear input and state constraints in [6]. The current paper provides a detailed treatment of the input and state constrained case [6] and discusses the line search algorithm in the presence of degenerate solutions.

In the presence of state and input constraints, the solution to the subproblems associated with individual stages of the multi-stage min-max optimization may, as a result of linearly dependent constraints, be degenerate. This results in the associated optimal costs being discontinuous functions of the state, making the problem significantly more challenging than the input-constrained problem considered in [7]. Degenerate subproblems, on the other hand, are highly relevant in practice, e.g. in aerospace applications which are governed by non-minimum phase dynamics (as discussed in Example 1 of Section 9). A first basic treatment of degenerate subproblems was provided in [6], however a theoretical framework for convergence of the active set algorithm and the relationship of a degenerate solution to the globally optimal solution was not presented.

The current paper proposes a method of handling linearly dependent constraints by circumventing the possibility of discontinuous optimal control laws (see e.g. [20]) and by selecting the active set changes that lead to continuity of the primal and dual variables along the line-of-search. This is the basis of a guarantee of convergence of the active set algorithm. We also relate the candidate solutions derived from first order necessary conditions in the presence of degenerate subproblems to the global solution of the dynamic programming problem. Finally, we establish a specified disturbance $l_{2}$-gain under the receding horizon application of the min-max optimal control law, which follows from the $\mathcal{H}_{\infty}$ performance index employed in this paper. Numerical examples demonstrate significant advantages in terms of computation and performance over the affine disturbance feedback strategy of [12].

\section{PROBLEM STATEMENT}

In this paper we are concerned with linear discrete time systems subjected to bounded disturbances:

$$
x_{t+1}=A x_{t}+B u_{t}+D w_{t}, \quad t=0,1, \ldots
$$

with state $x_{t} \in \mathbb{R}^{n_{x}}$, control input $u_{t} \in \mathbb{R}^{n_{u}}$ and disturbance input $w_{t} \in \mathbb{R}^{n_{w}}$. The matrices $A, B, D$ are assumed to be constant and known, and variables $x_{t}, u_{t}, w_{t}$ are subject to constraints: $x_{t} \in \mathcal{X}$, $u_{t} \in \mathcal{U}, w_{t} \in \mathcal{W}$, where $\mathcal{X}, \mathcal{U}, \mathcal{W}$ are compact and convex polytopic sets that contain the origin. 
We consider the following dynamic programming formulation of a constrained, finite horizon robust optimal control problem (see e.g. $[1,20])$ :

$$
\left(u_{m}^{*}(x), w_{m}^{*}(x, u)\right)=\arg \min _{u \in \mathcal{U}} \max _{w \in \mathcal{W}} J_{m}(x, u, w)
$$

subject to $A x+B u \in \mathcal{X}_{m-1} \ominus D \mathcal{W}$ ( $\ominus$ denotes Pontryagin difference), where the dynamic programming recursion on $J_{m}$ and $\mathcal{X}_{m}$ is defined for $m=1,2 \ldots$ by

$$
\begin{aligned}
& J_{m}(x, u, w)=\frac{1}{2}\left(\|x\|_{Q}^{2}+\|u\|_{R}^{2}-\gamma^{2}\|w\|^{2}\right)+J_{m-1}^{*}\left(x^{+}\right) \\
& J_{m}^{*}(x)=J_{m}\left(x, u_{m}^{*}(x), w_{m}^{*}\left(x, u_{m}^{*}(x)\right)\right) \\
& x^{+}=A x+B u+D w \\
& \mathcal{X}_{m}=\mathcal{X} \cap\left\{x: \exists u \in \mathcal{U}, A x+B u \in \mathcal{X}_{m-1} \ominus D \mathcal{W}\right\}
\end{aligned}
$$

with the terminal conditions:

$$
\begin{aligned}
J_{0}^{*}(x) & =\frac{1}{2}\|x\|_{P_{0}}^{2}, \\
\mathcal{X}_{0} & =\mathcal{X}^{f} .
\end{aligned}
$$

Here $x^{+}$denotes the successor state given $x, u$ and $w$, and $J_{m}^{*}(\cdot), u_{m}^{*}(\cdot)$ and $w_{m}^{*}(\cdot, \cdot)$ denote respectively the optimal cost, control input, and disturbance input for the $m$-stage problem defined in (2a). The corresponding receding horizon control law for a prediction horizon of $N$ time steps is given by $u_{t}=u_{N}^{*}\left(x_{t}\right)$.

In (2), $R$ is a positive-definite matrix (denoted $R \succ 0$ ), $Q$ is a positive semidefinite matrix (denoted $Q \succeq 0$ ) and $\|x\|_{Q}^{2}$ denotes $x^{T} Q x$. We assume that $P_{0}$ in (2f) is chosen so that $\left\|x_{0}\right\|_{P_{0}}^{2}=$ $\sum_{k=0}^{\infty}\left(\left\|x_{t}\right\|_{Q}^{2}+\left\|u_{t}\right\|_{R}^{2}-\gamma^{2}\left\|w_{t}\right\|^{2}\right)$ with $u_{t}=u^{f}\left(x_{t}\right)$ and $w_{t}=w^{f}\left(x_{t}, u_{t}\right)$, where $u^{f}(\cdot)$ and $w^{f}(\cdot, \cdot)$ are the optimal solutions of (2a-d) in the limit as $m \rightarrow \infty$ and in the absence of the constraints $x \in \mathcal{X}, u \in \mathcal{U}, w \in \mathcal{W}$. Section 4 derives conditions on the value of $\gamma$ (which is a design parameter); here we note that $\gamma$ must be large enough to ensure the existence of the solutions $u^{f}(\cdot), w^{f}(\cdot, \cdot)$ to the unconstrained $\mathcal{H}_{\infty}$ optimal control problem [20]. We assume that the initial state of (1) satisfies $x_{0} \in \mathcal{X}_{N}$.

\section{Remark 2.1}

The problem formulated in (2) defines a closed loop optimal control problem (e.g. [18]), since the optimal control law $u_{m}^{*}(\cdot)$ depends on the plant state $x$, while the worst-case disturbance $w_{m}^{*}(\cdot, \cdot)$ depends on the current control input $u$ as well as $x$. The sequential nature of this minmax problem and the fact that the optimization is performed over a set of arbitrary feedback laws $\left\{u_{m}(x), w_{m}(x, u), m=1, \ldots, N\right\}$ imply that, unlike open-loop formulations of robust MPC, Problem (2) cannot be solved for given $x_{0}$ as a single quadratic programming problem. The relationship between open and closed loop optimal control formulations based on $\mathcal{H}_{\infty}$-type costs is discussed in [7].

We denote predicted sequences of minimizing control inputs and maximizing disturbance inputs in (2) as $\left\{u_{0}, \ldots, u_{N-1}\right\}$ and $\left\{w_{0}, \ldots, w_{N-1}\right\}$, and the corresponding predicted state sequence as $\left\{x_{0}, \ldots, x_{N-1}\right\}$, so that $u_{k}=u_{N-k}^{*}\left(x_{k}\right), w_{k}=w_{N-k}^{*}\left(x_{k}, u_{k}\right)$ and $x_{k+1}=A x_{k}+B u_{k}+D w_{k}$ for $k=0, \ldots, N-1$. To differentiate between actual and predicted plant states, we denote the actual plant state, which is assumed to be known at time $t$, as $x^{p}$, and its value at time $t$ as $x_{t}^{p}$.

\section{SOLUTION OUTLINE}

Section 4 presents a novel derivation of the Karush-Kuhn-Tucker (KKT) conditions providing firstorder necessary conditions for optimality for Problem (2) (see e.g. [11]). For a given active set the corresponding equality constrained problem is solved using Riccati recursions (Section 5), using a sweep method (as in [5]) and results in a sequence of optimal state feedback control laws and 
worst-case disturbance functions. In Section 6 the equality constrained control policy is related to the inequality constrained Problem (2). Finally, a detailed discussion of the proposed active set method and its theoretical properties is provided in Section 7. In all sections a particular focus is on the unified treatment of: (a) the special case that the state and input constraints are linearly independent and necessary conditions are also sufficient (leading to unique solutions for given $x_{0}$ ); (b) the general case of degenerate constraints in which the necessary conditions can be used to obtain locally optimal solutions for the given plant state. A consistent treatment of both cases is obtained through the formulation of a line search algorithm based on homotopy of solutions (as in $[8,7]$ ).

\section{FIRST-ORDER NECESSARY CONDITIONS}

This section derives the first order necessary conditions for optimality by considering the relevant Lagrangian function at a given stage. We subsequently derive the linking conditions on the co-states between successive stages of the optimal control problem using a backwards induction procedure. We present a derivation of the first order conditions which, unlike the derivation in [6], does not rely on the Wolfe dual. Consequently this derivation applies to the general case of (possibly) discontinuous objective functions that result from degenerate subproblems of Problem (2).

Let the polytopic sets $\mathcal{X}_{m-1} \ominus D \mathcal{W}, \mathcal{U}$ and $\mathcal{W}$ be expressed as:

$$
\begin{aligned}
\mathcal{X}_{m-1} \ominus D \mathcal{W} & =\left\{x \in \mathbb{R}^{n_{x}}: E_{m} x \leq \mathbf{1}\right\} \\
\mathcal{U} & =\left\{u \in \mathbb{R}^{n_{u}}: F u \leq \mathbf{1}\right\} \\
\mathcal{W} & =\left\{w \in \mathbb{R}^{n_{w}}: G w \leq \mathbf{1}\right\}
\end{aligned}
$$

for $E_{m} \in \mathbb{R}^{n_{E} \times n_{x}}, F \in \mathbb{R}^{n^{f} \times n_{u}}, G \in \mathbb{R}^{n_{G} \times n_{w}}$, where $\mathbf{1}=[1 \cdots 1]^{T}$ denotes a vector of conformal dimensions. Also let $\lambda$ and $\hat{\lambda}$ denote the Lagrange multipliers associated with constraints $x^{+}=$ $\hat{x}+D w$ and $\hat{x}=A x+B u$, and let $\nu, \mu$ and $\eta$ denote the Lagrange multipliers for inequality constraints $\hat{x} \in \mathcal{X}_{m-1} \ominus D \mathcal{W}, u \in \mathcal{U}$ and $w \in \mathcal{W}$ respectively ${ }^{\dagger}$. Since the min-max subproblem (2a) at each stage may be degenerate due to linear dependence in the active constraint set, we include equality state constraints $C_{m} \hat{x}=1$ and $\hat{C}_{m} x^{+}=-1$ in the minimization and maximization $\left(\hat{C}_{m} \in \mathbb{R}^{n_{d_{m-1}} \times n_{x}}\right.$ and $\left.C_{m} \in \mathbb{R}^{n_{\hat{d}_{m}} \times n_{x}}\right)$ with associated multipliers $\zeta$ and $\hat{\zeta}$. These constraints are required for the equality problem (EP) solution as detailed in Section 5 and are therefore required to determine the region in state space within which the EP solution is primal/dual feasible for the inequality constrained problem. However, they are not actual constraints in the inequality constrained DP problem; i.e. they occur as a consequence of obtaining a solution candidate for the DP for a given degenerate active set.

The $m$-stage min-max optimization problem (2) is equivalent to the following sequence of subproblems

$$
J_{m}^{*}(x)=\min _{u, \hat{x}} \frac{1}{2}\|x\|_{Q}^{2}+\frac{1}{2}\|u\|_{R}^{2}+\hat{J}_{m}^{*}(\hat{x}) \quad \text { subject to } \quad\left\{\begin{array}{l}
E_{m} \hat{x} \leq \mathbf{1}, F u \leq \mathbf{1}, C_{m} \hat{x}=\mathbf{1} \\
\hat{x}=A x+B u
\end{array}\right.
$$

and

$$
\hat{J}_{m}^{*}(\hat{x})=\max _{w, x^{+}}-\frac{\gamma^{2}}{2}\|w\|^{2}+J_{m-1}^{*}\left(x^{+}\right) \quad \text { subject to } \quad\left\{\begin{array}{l}
G w \leq \mathbf{1}, \hat{C}_{m} x^{+}=-\mathbf{1} \\
x^{+}=\hat{x}+D w,
\end{array}\right.
$$

with $J_{0}^{*}(x)=\frac{1}{2}\|x\|_{P_{0}}^{2}$.

Correspondingly we define the associated Lagrangian functions:

\footnotetext{
${ }^{\dagger}$ Note that in this section we remove the time indices on the optimization variables for readibility
} 


$$
\begin{array}{r}
L_{m}(x, u, \mu, \nu, \hat{\zeta}, \hat{\lambda}, \hat{x})=\frac{1}{2}\|x\|_{Q}^{2}+\frac{1}{2}\|u\|_{R}^{2}+\hat{J}_{m}^{*}(\hat{x})-\mu^{T}(\mathbf{1}-F u)-\nu^{T}\left(\mathbf{1}-E_{m} \hat{x}\right) \\
-\hat{\zeta}^{T}\left(\mathbf{1}-C_{m} \hat{x}\right)-\hat{\lambda}^{T}(\hat{x}-(A x+B u))
\end{array}
$$

and

$$
\begin{aligned}
\hat{L}_{m}\left(\hat{x}, w, \eta, \zeta, \lambda, x^{+}\right)=-\frac{\gamma^{2}}{2}\|w\|^{2}+J_{m-1}^{*}\left(x^{+}\right)+\eta^{T}(\mathbf{1}-G w) & +\zeta^{T}\left(\mathbf{1}+\hat{C}_{m} x^{+}\right) \\
& -\lambda^{T}\left(x^{+}-(\hat{x}+D w)\right) .
\end{aligned}
$$

Then the first order necessary conditions for optimality of Problem (6b) are given by the KKT conditions (e.g. [11]):

$$
\begin{array}{lll}
\nabla_{w} \hat{L}_{m}=0 & \Longrightarrow & \gamma^{2} w+G^{T} \eta-D^{T} \lambda=0 \\
\nabla_{\zeta} \hat{L}_{m}=0 & \Longrightarrow & \hat{C}_{m} x^{+}=-\mathbf{1} \\
\nabla_{\lambda} \hat{L}_{m}=0 & \Longrightarrow & x^{+}=\hat{x}+D w \\
\nabla_{x^{+}} \hat{L}_{m}=0 & \Longrightarrow & \lambda=\nabla_{x^{+}} J_{m-1}^{*}\left(x^{+}\right)+\hat{C}_{m}^{T} \zeta
\end{array}
$$

and

$$
\eta^{T}(\mathbf{1}-G w)=0, \quad \eta \geq 0, \quad G w \leq \mathbf{1} .
$$

The first order necessary conditions for optimality of Problem (6a) are given similarly by

$$
\begin{aligned}
& \nabla_{u} L_{m}=0 \quad \Longrightarrow \quad R u+F^{T} \mu+B^{T} \hat{\lambda}=0 \\
& \nabla_{\hat{\zeta}} L_{m}=0 \quad \Longrightarrow \quad C_{m} \hat{x}=\mathbf{1} \\
& \nabla_{\hat{\lambda}} L_{m}=0 \quad \Longrightarrow \quad \hat{x}=A x+B u \\
& \nabla_{\hat{x}} L_{m}=0 \quad \Longrightarrow \quad \hat{\lambda}=\nabla_{\hat{x}} \hat{J}_{m}^{*}(\hat{x})+E_{m}^{T} \nu+C_{m}^{T} \hat{\zeta}
\end{aligned}
$$

and

$$
\begin{array}{ll}
\mu^{T}(\mathbf{1}-F u)=0, & \mu \geq 0, \quad F u \leq \mathbf{1}, \\
\nu^{T}\left(\mathbf{1}-E_{m} \hat{x}\right)=0, \quad \nu \geq 0, & E_{m} \hat{x} \leq \mathbf{1} .
\end{array}
$$

Lemma 4.1

Equations (8d) and (9d) are respectively equivalent to

$$
\begin{aligned}
& \lambda= \begin{cases}A^{T} \hat{\lambda}^{+}+Q x^{+}+\hat{C}_{m}^{T} \zeta, & \text { if } m>1 \\
P_{0} x^{+}+\hat{C}_{m}^{T} \zeta, & \text { if } m=1\end{cases} \\
& \hat{\lambda}=\lambda+E_{m}^{T} \nu+C_{m}^{T} \hat{\zeta},
\end{aligned}
$$

where $\hat{\lambda}^{+}$denotes the multiplier associated with the constraint $\hat{x}=A x+B u$ in the $(m-1)$-stagesto-go maximization problem.

Proof

Let $w^{*}(\hat{x}), \eta^{*}(\hat{x}), \zeta^{*}(\hat{x}), \lambda^{*}(\hat{x}), x^{+^{*}}(\hat{x})$, denote the optimal values of primal variables and multipliers for Problem (6b) for given $\hat{x}$. Then, using (8a-d), we have

$$
\begin{aligned}
\nabla_{\hat{x}} \hat{J}_{m}^{*}(\hat{x}) & =\nabla_{\hat{x}} \hat{L}_{m}\left(\hat{x}, w^{*}(\hat{x}), \eta^{*}(\hat{x}), \zeta^{*}(\hat{x}), \lambda^{*}(\hat{x}), x^{+^{*}}(\hat{x})\right) \\
& =\frac{\partial \hat{L}_{m}}{\partial \hat{x}}+\frac{\partial w^{*}}{\partial \hat{x}} \nabla_{w} \hat{L}_{m}+\cdots+\frac{\partial x^{+^{*}}}{\partial \hat{x}} \nabla_{x^{+}} \hat{L}_{m} \\
& =\lambda^{*}(\hat{x}) .
\end{aligned}
$$


Similarly denoting the optimal primal variables and multipliers for Problem (6a) for given $x$ as $u^{*}(x), \mu^{*}(x), \nu^{*}(x), \hat{\zeta}^{*}(x), \hat{\lambda}^{*}(x), \hat{x}^{*}(x)$, and using (9a-d), we also have

$$
\begin{aligned}
\nabla_{x} J_{m}^{*}(x) & =\nabla_{x} L_{m}\left(x, u^{*}(x), \mu^{*}(x), \nu^{*}(x), \hat{\zeta}^{*}(x), \hat{\lambda}^{*}(x), \hat{x}^{*}(x)\right) \\
& =\frac{\partial L_{m}}{\partial x}+\frac{\partial u^{*}}{\partial x} \nabla_{u} L_{m}+\cdots+\frac{\partial \hat{x}^{*}}{\partial x} \nabla_{\hat{x}} L_{m} \\
& =A^{T} \hat{\lambda}^{*}(x)+Q x .
\end{aligned}
$$

so that $\nabla_{x^{+}} J_{m-1}^{*}\left(x^{+}\right)=A^{T} \hat{\lambda}^{+}+Q x^{+}$if $m>1$, and $\nabla_{x^{+}} J_{m-1}^{*}\left(x^{+}\right)=P_{0} x^{+}$if $m=1$, which implies $(10 \mathrm{a}, \mathrm{b})$.

\section{Remark 4.2}

The optimal values of the decision variables $\left(u^{*}(x), \hat{x}^{*}(x)\right)$ and $\left(w^{*}(\hat{x}), x^{+*}(\hat{x})\right)$ are in general multivalued functions of their arguments, $x$ and $\hat{x}$, respectively. Hence $J_{m}^{*}(x)$ and $\hat{J}_{m}^{*}(\hat{x})$ are also multivalued in general. This situation occurs as a result of linear dependence in the active constraint set at a given minimization stage. This has the effect that the gradient of the optimal value function at the given stage is discontinuous in the given parameter and causes the optimization problems at prior stages to be non-convex-concave, in general. The focus of the proposed active set method is to handle both non-degenerate and degenerate subproblems by using a selection that enables the gradients introduced in the proof of Lemma 1 to be defined uniquely along the line of search.

\section{THE ACTIVE SET EQUALITY CONSTRAINT PROBLEM}

Let $\mathcal{A}_{m}=\left\{\mathcal{A}_{m}^{u}, \mathcal{A}_{m}^{\hat{x}}, \mathcal{A}_{m}^{w}\right\}$ denote the set of indices of the active constraints in the $m$-stages-togo minimization and maximization problems in $(6 a, b)$, so that the solution for primal variables $u^{*}, w^{*}, \hat{x}^{*}$ and dual variables $\mu^{*}, \nu^{*}, \eta^{*}$, satisfies

$$
\begin{array}{lll}
e_{i}^{T} F u^{*}=1, \quad \forall i \in \mathcal{A}_{m}^{u}, & e_{i}^{T} \mu^{*}=0, \quad \forall i \notin \mathcal{A}_{m}^{u} \\
e_{i}^{T} E_{m} \hat{x}^{*}=1, \quad \forall i \in \mathcal{A}_{m}^{\hat{x}}, & e_{i}^{T} \nu^{*}=0, \quad \forall i \notin \mathcal{A}_{m}^{\hat{x}} \\
e_{i}^{T} G w^{*}=1, \quad \forall i \in \mathcal{A}_{m}^{w}, & e_{i}^{T} \eta^{*}=0, \quad \forall i \notin \mathcal{A}_{m}^{w}
\end{array}
$$

where $e_{i}$ is the $i$ th column of an identity matrix of conformal dimensions. To simplify notation, for a given active set $\mathcal{A}_{m}$ we define $F_{m}, G_{m}$, and redefine $E_{m}$ so that the active constraints of (13a-c) are equivalent to:

$$
F_{m} u=1, \quad E_{m} \hat{x}=1, \quad G_{m} w=1,
$$

and similarly we redefine $\mu, \nu$ and $\eta$ as the vectors of multipliers of the active constraints indexed by $\mathcal{A}_{m}^{u}, \mathcal{A}_{m}^{\hat{x}}$ and $\mathcal{A}_{m}^{w}$ respectively. To ensure full generality we assume that the active constraints at each stage $m \geq 1$ may be linearly dependent. In addition we assume for notational convenience that the equality constraints $\hat{C}_{m} x^{+}=-\mathbf{1}$ and $C_{m} \hat{x}=\mathbf{1}$ appearing in (6a,b) are the compatibility conditions associated with linearly dependent constraints. We illustrate the algebraic details of the solution to non-degenerate and degenerate subproblems separately. We first consider the single-stage problem to demonstrate the algebraic conditions for degeneracy. In a second step the general multi-stage problem is discussed. Finally these results are summarized in Theorem 5.1.

\subsection{Single-stage problem}

For the equality problem (EP) associated with active set $\mathcal{A}_{1}^{w}$ in the maximization (6b) at $m=1$ we have, from (10a)

$$
\lambda=P_{0} x^{+} .
$$

Eliminating $\lambda$ and $x^{+}$from (8a-c) and invoking $G_{1} w=\mathbf{1}$ therefore gives

$$
\left[\begin{array}{cc}
\gamma^{2} I-D^{T} P_{0} D & G_{1}^{T} \\
G_{1} & 0
\end{array}\right]\left[\begin{array}{l}
w \\
\eta
\end{array}\right]=\left[\begin{array}{c}
D^{T} P_{0} \\
0
\end{array}\right] \hat{x}+\left[\begin{array}{l}
0 \\
\mathbf{1}
\end{array}\right] .
$$


The solution of (16) can be expressed as

$$
\left[\begin{array}{c}
w \\
\eta
\end{array}\right]=\Psi_{1}\left[\begin{array}{c}
D^{T} P_{0} \\
0
\end{array}\right] \hat{x}+\Psi_{1}\left[\begin{array}{l}
0 \\
1
\end{array}\right]
$$

for some matrix $\Psi_{1}$ where it has been assumed that $G_{1, \perp}^{T}\left(\gamma^{2} I-D^{T} P_{0} D\right) G_{1, \perp}$ is non-singular. Details on how to compute $\Psi_{1}$ are given in [11], Section 10.2.

For the EP with active set $\left\{\mathcal{A}_{1}^{\hat{x}}, \mathcal{A}_{1}^{u}\right\}$ in the minimization (6a) at $m=1,(15),(10 \mathrm{~b})$ and (17) imply

$$
\begin{gathered}
\hat{\lambda}=\hat{P}_{1} \hat{x}+\hat{q}_{1}+E_{1}^{T} \nu \\
{\left[\begin{array}{ll}
\hat{P}_{1} & \hat{q}_{1}
\end{array}\right]=\left[\begin{array}{ll}
P_{0} & 0
\end{array}\right]+\left[\begin{array}{ll}
P_{0} D & 0
\end{array}\right] \Psi_{1}\left[\begin{array}{cc}
D^{T} P_{0} & 0 \\
0 & \mathbf{1}
\end{array}\right] .}
\end{gathered}
$$

By eliminating $\hat{\lambda}$ and $\hat{x}$ from (9a-c) and invoking $E_{1} \hat{x}=\mathbf{1}$ and $F_{1} u=\mathbf{1}$, we therefore obtain

$$
\left[\begin{array}{ccc}
R+B^{T} \hat{P}_{1} B & B^{T} E_{1}^{T} & F_{1}^{T} \\
E_{1} B & 0 & 0 \\
F_{1} & 0 & 0
\end{array}\right]\left[\begin{array}{l}
u \\
\nu \\
\mu
\end{array}\right]=-\left[\begin{array}{c}
B^{T} \hat{P}_{1} \\
E_{1} \\
0
\end{array}\right] A x+\left[\begin{array}{c}
-B^{T} \hat{q}_{1} \\
\mathbf{1} \\
\mathbf{1}
\end{array}\right]
$$

Assuming that $R+B^{T} \hat{P}_{0} B$ is non-singular, the general solution of (19) (which includes the degenerate case in which the columns of $\left[\begin{array}{lll}B^{T} & E_{1}^{T} & F_{1}^{T}\end{array}\right]$ are linearly dependent) can be expressed as

$$
\left[\begin{array}{l}
u \\
\nu \\
\mu
\end{array}\right]=-\Theta_{1}\left[\begin{array}{c}
B^{T} \hat{P}_{1} \\
E_{1} \\
0
\end{array}\right] A x+\Theta_{1}\left[\begin{array}{c}
-B^{T} \hat{q}_{1} \\
\mathbf{1} \\
\mathbf{1}
\end{array}\right]-\left[\begin{array}{c}
0 \\
Z_{1,1} \\
Z_{2,1}
\end{array}\right] \beta .
$$

for some matrix $\Theta_{1}$. Details on how to compute $\Theta_{1}$ are given in [11], Section 10.2. In the degenerate case, $\beta$ contains the free variables in the solution of (19), and $Z_{1}=\left[Z_{1,1}^{T} Z_{2,1}^{T}\right]^{T}$ is the full-rank matrix satisfying

$$
Z_{1}^{T}\left[\begin{array}{c}
E_{1} B \\
F_{1}
\end{array}\right]=0, \quad Z_{1}^{T}\left[\begin{array}{l}
\mathbf{1} \\
\mathbf{1}
\end{array}\right]=\mathbf{1} .
$$

For the non-degenerate case the columns of $\left[B^{T} E_{1}^{T} F_{1}^{T}\right]$ are linearly independent and we set $Z_{1}=0$ and $\beta=0$. In the degenerate case, (19) admits solutions if and only if $x$ satisfies the compatibility condition

$$
\hat{C}_{2} x=-\mathbf{1}, \text { where } \hat{C}_{2}=-Z_{1}^{T} E_{1} A .
$$

Thus (21) constitutes an equality constraint in the maximization problem (6b) at $m=2$, and (18), (20) and (21) imply

$$
\begin{gathered}
A^{T} \hat{\lambda}+Q x=P_{1} x+q_{1}+\hat{C}_{2}^{T} \beta \\
{\left[\begin{array}{ll}
P_{1} & q_{1}
\end{array}\right]=\left[\begin{array}{llll}
Q+A^{T} \hat{P}_{1} A & A^{T} \hat{q}_{1}
\end{array}\right]+A^{T}\left[\begin{array}{lll}
\hat{P}_{1} B & E_{1}^{T} & 0
\end{array}\right] \Theta_{1}\left[\begin{array}{cc}
-B^{T} \hat{P}_{1} A & -B^{T} \hat{q}_{1} \\
-E_{1} A & \mathbf{1} \\
0 & \mathbf{1}
\end{array}\right] .}
\end{gathered}
$$

\subsection{Multi-stage problem}

Consider now the EP at stage $m>1$ with active set $\mathcal{A}_{m}$. If the minimization problem (6a) at stage $m-1$ is degenerate due to linearly dependent constraints, then its solution contains free variables, denoted $\beta^{+}$in (23), which can be determined by solving the maximization problem (6b) at stage $m$ as we now discuss. From (10a) and (22a) we have

$$
\lambda=P_{m-1} x^{+}+q_{m-1}+\hat{C}_{m}^{T}\left(\zeta+\beta^{+}\right) .
$$

By eliminating $\lambda$ and $x^{+}$from (8a-c) and using $G_{m} w=1$ we obtain

$$
\left[\begin{array}{ccc}
\gamma^{2} I-D^{T} P_{m-1} D & -D^{T} \hat{C}_{m}^{T} & G_{m}^{T} \\
-\hat{C}_{m} D & 0 & 0 \\
G_{m} & 0 & 0
\end{array}\right]\left[\begin{array}{c}
w \\
\zeta+\beta^{+} \\
\eta
\end{array}\right]=\left[\begin{array}{c}
D^{T} P_{m-1} \\
\hat{C}_{m} \\
0
\end{array}\right] \hat{x}+\left[\begin{array}{c}
D^{T} q_{m-1} \\
\mathbf{1} \\
\mathbf{1}
\end{array}\right]
$$


The general solution of (24) can be expressed as

$$
\begin{aligned}
{\left[\begin{array}{c}
w \\
\zeta+\beta^{+} \\
\eta
\end{array}\right] } & =\Psi_{m}\left[\begin{array}{c}
D^{T} P_{m-1} \\
\hat{C}_{m} \\
0
\end{array}\right] \hat{x}+\Psi_{m}\left[\begin{array}{c}
D^{T} q_{m-1} \\
\mathbf{1} \\
\mathbf{1}
\end{array}\right]-\left[\begin{array}{c}
0 \\
\hat{Z}_{1, m} \\
\hat{Z}_{2, m}
\end{array}\right] \hat{\beta} \\
& =\left[\begin{array}{c}
M_{m}^{w} \\
M_{m}^{\zeta+\beta^{+}} \\
M_{m}^{\eta}
\end{array}\right] \hat{x}+\left[\begin{array}{c}
m_{m}^{w} \\
m_{m}^{\zeta+\beta^{+}} \\
m_{m}^{\eta}
\end{array}\right]-\left[\begin{array}{c}
0 \\
\hat{Z}_{1, m} \\
\hat{Z}_{2, m}
\end{array}\right] \hat{\beta}
\end{aligned}
$$

For the case that (24) is degenerate (i.e. the columns of $\left[-D^{T} \hat{C}_{m}^{T} G_{m}^{T}\right]$ are linearly dependent), $\hat{\beta}$ contains the free variables in the solution of (24) and $\hat{Z}_{m}=\left[\begin{array}{lll}\hat{Z}_{1, m}^{T} & \hat{Z}_{2, m}^{T}\end{array}\right]^{T}$ is the full-rank matrix satisfying

$$
\hat{Z}_{m}^{T}\left[\begin{array}{c}
-\hat{C}_{m} D \\
G_{m}
\end{array}\right]=0, \quad \hat{Z}_{m}^{T}\left[\begin{array}{l}
\mathbf{1} \\
\mathbf{1}
\end{array}\right]=\mathbf{1}
$$

whereas $\hat{Z}_{m}=0, \hat{\beta}=0$ if (24) is non-degenerate. In the degenerate case, $\hat{x}$ must satisfy the compatibility condition given by the following constraint in the minimization (6a) at stage $m$ :

$$
C_{m} \hat{x}=\mathbf{1}, \text { where } C_{m}=-\hat{Z}_{1, m}^{T} \hat{C}_{m}
$$

Therefore (23), (10b) and (25) imply

$$
\hat{\lambda}=\hat{P}_{m} \hat{x}+\hat{q}_{m}+E_{m}^{T} \nu+C_{m}^{T}(\hat{\zeta}+\hat{\beta})
$$

where

$$
\left[\begin{array}{ll}
\hat{P}_{m} & \hat{q}_{m}
\end{array}\right]=\left[\begin{array}{ll}
P_{m-1} & q_{m-1}
\end{array}\right]+\left[\begin{array}{lll}
P_{m-1} D & \hat{C}_{m}^{T} & 0
\end{array}\right] \Psi_{m}\left[\begin{array}{cc}
D^{T} P_{m-1} & D^{T} q_{m-1} \\
\hat{C}_{m} & \mathbf{1} \\
0 & \mathbf{1}
\end{array}\right] .
$$

Eliminating $\hat{\lambda}$ and $\hat{x}$ from (9a-c) and invoking $E_{m} \hat{x}=\mathbf{1}$ and $F_{m} u=\mathbf{1}$ gives

$$
\left[\begin{array}{cccc}
R+B^{T} \hat{P}_{m} B & B^{T} C_{m}^{T} & B^{T} E_{m}^{T} & F_{m}^{T} \\
C_{m} B & 0 & 0 & 0 \\
E_{m} B & 0 & 0 & 0 \\
F_{m} & 0 & 0 & 0
\end{array}\right]\left[\begin{array}{c}
u \\
\hat{\zeta}+\hat{\beta} \\
\nu \\
\mu
\end{array}\right]=-\left[\begin{array}{c}
B^{T} \hat{P}_{m} \\
C_{m} \\
E_{m} \\
0
\end{array}\right] A x+\left[\begin{array}{c}
-B^{T} \hat{q}_{m} \\
\mathbf{1} \\
\mathbf{1} \\
\mathbf{1}
\end{array}\right]
$$

The general solution of (29) can be expressed as

$$
\begin{aligned}
{\left[\begin{array}{c}
u \\
\hat{\zeta}+\hat{\beta} \\
\nu \\
\mu
\end{array}\right]=-\Theta_{m}\left[\begin{array}{c}
B^{T} \hat{P}_{m} \\
C_{m} \\
E_{m} \\
0
\end{array}\right] A x+\Theta_{m}\left[\begin{array}{c}
-B^{T} \hat{q}_{m} \\
\mathbf{1} \\
\mathbf{1} \\
\mathbf{1}
\end{array}\right]-\left[\begin{array}{c}
0 \\
Z_{1, m} \\
Z_{2, m} \\
Z_{3, m}
\end{array}\right] \beta } \\
=\left[\begin{array}{c}
L_{m}^{u} \\
L_{m}^{\hat{\zeta}+\hat{\beta}} \\
L_{m}^{\nu} \\
L_{m}^{\mu}
\end{array}\right] x+\left[\begin{array}{c}
l_{m}^{u} \\
\hat{l}_{m}^{\hat{s}+\hat{\beta}} \\
l_{m}^{\nu} \\
l_{m}^{\mu}
\end{array}\right]-\left[\begin{array}{c}
0 \\
Z_{1, m} \\
Z_{2, m} \\
Z_{3, m}
\end{array}\right] \beta
\end{aligned}
$$

For the degenerate case in which the columns of the matrix $\left[\begin{array}{lll}B^{T} C_{m}^{T} & B^{T} E_{m}^{T} & F_{m}^{T}\end{array}\right]$ are linearly dependent, $\beta$ contains the free variables in the solution of (29) and $Z_{m}=\left[Z_{1, m}^{T} Z_{2, m}^{T} Z_{3, m}^{T}\right]^{T}$ is the full-rank matrix satisfying

$$
Z_{m}^{T}\left[\begin{array}{c}
C_{m} B \\
E_{m} B \\
F_{m}
\end{array}\right]=0, \quad Z_{m}^{T}\left[\begin{array}{l}
\mathbf{1} \\
\mathbf{1} \\
\mathbf{1}
\end{array}\right]=\mathbf{1}
$$


whereas $Z_{m}=0$ and $\beta=0$ if (29) is non-degenerate. In the degenerate case (29) admits solutions if and only if $x$ satisfies the compatibility condition

$$
\hat{C}_{m+1} x=-\mathbf{1}, \text { where } \hat{C}_{m+1}=-\left[\begin{array}{ll}
Z_{1, m}^{T} & Z_{2, m}^{T}
\end{array}\right]\left[\begin{array}{l}
C_{m} \\
E_{m}
\end{array}\right] A .
$$

From (27), (30) and making use of (31) we therefore have

$$
\begin{aligned}
& A^{T} \hat{\lambda}+Q x=P_{m} x+q_{m}+\hat{C}_{m+1}^{T} \beta \\
& {\left[\begin{array}{ll}
P_{m} & q_{m}
\end{array}\right]=\left[\begin{array}{ll}
Q+A^{T} \hat{P}_{m} A & A^{T} \hat{q}_{m}
\end{array}\right]+A^{T}\left[\begin{array}{llll}
\hat{P}_{m} B & C_{m}^{T} & E_{m}^{T} & 0
\end{array}\right] \Theta_{m}\left[\begin{array}{cc}
-B^{T} \hat{P}_{m} A & -B^{T} \hat{q}_{m} \\
-C_{m} A & \mathbf{1} \\
-E_{m} A & \mathbf{1} \\
0 & \mathbf{1}
\end{array}\right]}
\end{aligned}
$$

The following theorem summarizes the Riccati recursion defined by the procedure described above for solving the min-max problems $(6 \mathrm{a}, \mathrm{b})$ corresponding to a given sequence of active sets $\mathcal{A}=\left\{\mathcal{A}_{1}, \ldots, \mathcal{A}_{N}\right\}$.

\section{Theorem 5.1}

The solutions of the EPs corresponding to the min-max problems (6a,b) with active set $\mathcal{A}_{m}=$ $\left\{\mathcal{A}_{m}^{u}, \mathcal{A}_{m}^{\hat{x}}, \mathcal{A}_{m}^{w}\right\}, u_{k}=u_{N-k}^{*}\left(x_{k}\right), w_{k}=w_{N-k}^{*}\left(x_{k}, u_{k}\right)$, are given by (25) and (30), where $\hat{P}_{m}, \hat{q}_{m}$, $P_{m}$ and $q_{m}$ are defined recursively by (28) and (32b) for $m=1, \ldots, N$.

Proof

This can be shown by induction on $\lambda$ in (23). Thus, for $m=1$ (23) reduces to (15), while for $m>1$, (10a) and (32b) imply that the multiplier $\lambda$ in (7b), with $m$ replaced by $m+1$, is given by (23) with $m$ replaced by $m+1$.

\section{OPTIMALITY OF INEQUALITY CONSTRAINED PROBLEM}

This section gives conditions for optimality of the Riccati recursion (25), (28) and (30), (32b) and, in particular, considers in detail the situation involving degenerate subproblems (in contrast to [6]). In general the KKT conditions admit non-unique solutions in the presence of degenerate constraints, since the corresponding Lagrange multipliers are non-unique and can thus cause the gradient of the optimal cost to be discontinuous. In the following we state the conditions for a local solution to Problem (2). Provided that there exists no degenerate subproblem at any given stage for a particular active set (at a given initial state), the solution to Problem (2) is uniquely given by the KKT conditions. If there exists a degenerate active set (at a given state), then more than one solution candidate generally exists. The non-degenerate solution candidate provides a locally optimal solution to Problem (2), whereas the degenerate solution candidate provides a locally optimal solution to Problem (2) on a subspace characterized by the necessary compatibility constraints.

Theorem 6.1

The functions $w_{m}^{*}(\hat{x}), u_{m}^{*}(x)$, defined in (25) and (30) for each stage $m=1, \ldots, N$ by

$$
\begin{aligned}
w_{m}^{*}(\hat{x}) & =M_{m}^{w} \hat{x}+m_{m}^{w} \\
u_{m}^{*}(x) & =L_{m}^{u} x+l_{m}^{u},
\end{aligned}
$$

are locally optimal solutions of Problem (2) if and only if: (i) the first-order necessary (KKT) conditions,

$$
\begin{gathered}
G\left(M_{m}^{w} \hat{x}_{k}+m_{m}^{w}\right) \leq 1 \\
F\left(L_{m}^{u} x_{k}+l^{u}\right) \leq 1, \quad E_{m} \hat{x}_{k} \leq 1
\end{gathered}
$$




$$
\begin{gathered}
M_{m}^{\eta} \hat{x}_{k}+m_{m}^{\eta}-\hat{Z}_{2, m} \hat{\beta}_{k} \geq 0 \\
L_{m}^{\nu} x_{k}+l_{m}^{\nu}-Z_{2, m} \beta_{k} \geq 0, \quad L_{m}^{\mu} x_{k}+l_{m}^{\mu}-Z_{3, m} \beta_{k} \geq 0,
\end{gathered}
$$

hold for $m=1, \ldots, N$ (with $k=N-m$ ); and (ii) the second-order sufficient conditions,

$$
\begin{aligned}
& {\left[\begin{array}{ll}
-D^{T} \hat{C}_{m}^{T} & G_{m}^{T}
\end{array}\right]_{\perp}^{T}\left(-\gamma^{2} I+D^{T} P_{m} D\right)\left[\begin{array}{c}
-\hat{C}_{m} D \\
G_{m}
\end{array}\right]_{\perp} \prec 0} \\
& {\left[\begin{array}{lll}
B^{T} C_{m}^{T} & B^{T} E_{m}^{T} & F_{m}^{T}
\end{array}\right]_{\perp}\left(R+B^{T} \hat{P}_{m} B\right)\left[\begin{array}{c}
C_{m} B \\
E_{m} B \\
F_{m}
\end{array}\right]_{\perp} \succ 0}
\end{aligned}
$$

hold for $m=1, \ldots, N$; and (iii) the compatibility conditions with $\zeta_{k}=0$ and $\hat{\zeta}_{k}=0$,

$$
\begin{aligned}
\beta_{k+1} & =M_{m}^{\zeta+\beta} \hat{x}_{k}+m_{m}^{\zeta+\beta}-\hat{Z}_{1, m} \hat{\beta}_{k} \\
\hat{\beta}_{k} & =L_{m}^{\hat{\zeta}+\hat{\beta}} x_{k}+l_{m}^{\hat{\zeta}+\hat{\beta}}-Z_{1, m} \beta_{k},
\end{aligned}
$$

hold for $m=2, \ldots, N$ (with $k=N-m$ ).

Proof

The first-order conditions (34a,b) follow from Theorem 5.1. For a given EP the second-order sufficient conditions as given in ([11], Theorem 9.3.2) lead to conditions $(35 \mathrm{a}, \mathrm{b})$ and ensure that $w_{m}^{*}, u_{m}^{*}$ are strict local solutions of Problem (2). In the case of degenerate subproblems, the EP solution provides a local solution to Problem (2) on a subspace defined by the compatibility constraints.

We note that if the minimization subproblem with $m=N$ is degenerate, then $\beta_{0}$ is a free variable, which may be determined as discussed in Section 7. On the other hand, the values of $\beta_{k+1}$ and $\hat{\beta}_{k}$ for $k=0, \ldots, N-2$ in degenerate subproblems at $m=N-k$ are determined as functions of $\hat{x}_{k}, \hat{\beta}_{k}$ and $x_{k}, \beta_{k}$ by (36a) and (36b) respectively.

\section{Theorem 6.2}

At a given $x_{0}$ let a solution candidate for Problem (2) corresponding to a sequence of non-degenerate active sets be given. Further assume that at $x_{0}$ no degenerate solution candidate to Problem (2) exists and that conditions $(35 \mathrm{a}, \mathrm{b})$ are satisfied. Then $(33 \mathrm{a}, \mathrm{b})$ are the unique solutions satisfying the KKT conditions of (8) and (9), and are the global optimizers of Problem (2).

Proof

This follows by induction on the DP recursion equations in (2) using the relevant optimal costs at a given stage, starting with $J_{0}^{*}(x)$. Specifically, from (12) and (11) and the uniqueness of the solutions of the EPs (25) and (30) for a non-degenerate active set, it follows that the optimal costs $\hat{J}_{m}^{*}(\hat{x})$ and $J_{m}^{*}(x)$ are continuously differentiable, and therefore the second-order conditions (35) ensure uniqueness and optimality of the solution $(33 \mathrm{a}, \mathrm{b})$ at stage $m+1$, for $m=0, \ldots, N-1$.

\section{Remark 6.3}

Although the choices of $\zeta$ and $\hat{\zeta}$ do not affect the EP primal solution, they do influence the dual solution. The choices $\zeta=0$ and $\hat{\zeta}=0$ ensure continuity of the dual solution at the boundaries of the region within which a specific active set satisfies the first order optimality conditions (whereas the primal solution is necessarily continuous). The elements of $\beta$ and $\hat{\beta}$ corresponding to the multipliers of new degenerate constraints are set to zero at the associated region boundary. Through these continuity-preserving definitions we are able to formulate an active set algorithm based on homotopy of solutions. The line-search proposed in Section 7 is guaranteed to recover a solution candidate to Problem (2), which is globally optimal provided that the conditions of Theorem 6.2 hold. Otherwise the solution is locally optimal with respect to Problem (2). 
Remark 6.4

Demonstrating the existence of an $l_{2}$-gain bound in closed loop requires special consideration (see Section 8), since, as discussed, the solution generated by the active set algorithm may not necessarily be globally optimal. One such case, as noted before, occurs in the presence of degenerate subproblems. A second situation occurs when conditions (35) fail to hold. In the case that at a particular stage $m$ the reduced Hessian in $(35 a)$ is indefinite, there exists a unique subspace $\mathcal{Q}^{+} \subseteq \mathcal{Q} \subseteq \mathbb{R}^{n_{w}}$ (where $\mathcal{Q}=\operatorname{span}\left[\begin{array}{c}-\hat{C}_{m} D \\ G_{m}\end{array}\right]_{\perp}$ ) in which $(35 a)$ is positive definite. Therefore the solution obtained (by the line-search) corresponds to a saddle point solution of the non-concave $w$-optimization subproblem at this stage.

\section{ACTIVE SET METHOD}

This section proposes a method of solving (2) for a given plant state $x^{p}$ using the Riccati recursion of Section 4. Here for convenience a forwards-in-time notation is used, i.e. $k$ denotes the time index (whereas $m=N-k$ denotes the number of stages-to-go). Starting from an initial state $x_{0} \neq x^{p}$ for which the optimal active set sequence $\mathcal{A}=\left\{\mathcal{A}_{N}, \ldots, \mathcal{A}_{1}\right\}$ is known (such as, for example, $x_{0}=0$, $\mathcal{A}=\{\emptyset, \ldots, \emptyset\}$ ), and using (25), (30) to determine optimal control, disturbance and multiplier sequences for (2) as functions of $x_{0}$, the approach uses a line-search in the space of $x_{0}$ to update the active set until $x_{0}=x^{p}$. We discuss continuity properties of solutions before describing the algorithm, its convergence and computation. In comparison to [6], we establish novel convergence results in the presence of degenerate subproblems. In particular, in order to ensure continuity of the optimal solution at (possibly overlapping) parametric programming regions, the active set solver requires a change in the line-search direction whenever a new degenerate constraint is added to or subtracted from the current active set.

In common with active set solvers employing floating point arithmetic, we make the following assumption.

Assumption 7.1

Only a single constraint can become active or inactive at each iteration.

Using (1) and (33), for given $x_{0}$ and active set $\mathcal{A}$ we obtain:

$$
x_{k}=\Phi_{k} x_{0}+\phi_{k}, \quad k=1, \ldots, N
$$

where $\Phi_{k}, \phi_{k}$ are defined by $\Phi_{0}=I, \phi_{0}=0$ and

$$
\begin{aligned}
\Phi_{k+1} & =\left(I+D M_{k}^{w}\right)\left(A+B L_{k}^{u}\right) \Phi_{k} \\
\phi_{k+1} & =\left(I+D M_{k}^{w}\right)\left(\left(A+B L_{k}^{u}\right) \phi_{k}+B l_{k}^{u}\right)+D m_{k}^{w} .
\end{aligned}
$$

Therefore $\left\{u_{0}, \ldots, u_{N-1}\right\}$ and $\left\{w_{0}, \ldots, w_{N-1}\right\}$ can be determined as affine functions of $x_{0}$, which we denote as $\mathbf{u}\left(x_{0}, \mathcal{A}\right)$ and $\mathbf{w}\left(x_{0}, \mathcal{A}\right)$, respectively. Similarly, from (25), (30), (36a) and (36b), the multiplier sequences $\left\{\mu_{0}, \ldots, \mu_{N-1}\right\},\left\{\nu_{0}, \ldots, \nu_{N-1}\right\}$ and $\left\{\eta_{0}, \ldots, \eta_{N-1}\right\}$ are obtained as affine functions of $x_{0}$, and (in the case that $\mathcal{A}$ contains a degenerate subproblem at $k=0$ ) $\beta_{0}$, which we denote as $\boldsymbol{\mu}\left(x_{0}, \beta_{0}, \mathcal{A}\right), \boldsymbol{\nu}\left(x_{0}, \beta_{0}, \mathcal{A}\right)$ and $\boldsymbol{\eta}\left(x_{0}, \beta_{0}, \mathcal{A}\right)$. Hence the collection, $\mathbf{p}$, of predicted sequences for given $x_{0}, \beta_{0}$ and $\mathcal{A}$ can be expressed

$$
\mathbf{p}\left(x_{0}, \beta_{0}, \mathcal{A}\right)=\left\{\mathbf{u}\left(x_{0}, \mathcal{A}\right), \mathbf{w}\left(x_{0}, \mathcal{A}\right), \boldsymbol{\mu}\left(x_{0}, \beta_{0}, \mathcal{A}\right), \boldsymbol{\nu}\left(x_{0}, \beta_{0}, \mathcal{A}\right), \boldsymbol{\eta}\left(x_{0}, \beta_{0}, \mathcal{A}\right)\right\} .
$$

Let $\mathcal{X}(\mathcal{A})$ denote the subset of the feasible initial conditions for (2) within which the EP solution $\mathbf{p}\left(x_{0}, \beta_{0}, \mathcal{A}\right)$ satisfies the optimality conditions of Theorem 6.1, namely

$$
\mathcal{X}(\mathcal{A})=\left\{x_{0} \in \mathcal{X}_{N}: \mathcal{A}^{*}\left(x_{0}\right)=\mathcal{A} \text { for some } \beta_{0}\right\} .
$$

Then clearly $\bigcup_{\mathcal{A} \in \Omega} \mathcal{X}(\mathcal{A})$, where $\Omega$ is the set of all possible active sets, must cover $\mathcal{X}_{N}$. Also by linearity of $(34 \mathrm{a}, \mathrm{b}),(36 \mathrm{a}),(36 \mathrm{~b})$ and $(37), \mathcal{X}(\mathcal{A})$ is a compact convex polytopic set. 
Lemma 7.2

If $\mathcal{A}^{1}$ is an active set that is not degenerate at $k=0$, so that $\mathbf{p}=\mathbf{p}\left(x_{0}, \mathcal{A}^{1}\right)$ for all $x_{0} \in \mathcal{X}\left(\mathcal{A}^{1}\right)$, then:

(i). For each $x_{0} \in \partial \mathcal{X}\left(\mathcal{A}^{1}\right)$ (where $\partial \mathcal{X}(\mathcal{A})$ is the boundary of $\mathcal{X}(\mathcal{A})$ ), $x_{0} \in \partial \mathcal{X}\left(\mathcal{A}^{2}\right)$ for a unique active set $\mathcal{A}^{2}$.

(ii). If $x_{0} \in \partial \mathcal{X}\left(\mathcal{A}^{1}\right) \cap \partial \mathcal{X}\left(\mathcal{A}^{2}\right)$, then $\mathbf{p}\left(x_{0}, \mathcal{A}^{1}\right)=\mathbf{p}\left(x_{0}, \mathcal{A}^{2}\right)$ if $\mathcal{A}^{2}$ is not degenerate at $k=0$, and $\mathbf{p}\left(x_{0}, \mathcal{A}^{1}\right)=\mathbf{p}\left(x_{0}, \beta_{0}, \mathcal{A}^{2}\right)$ for some $\beta_{0}$ otherwise.

If $\mathcal{A}^{1}$ is degenerate at $k=0$, then:

(iii). $\mathcal{X}\left(\mathcal{A}^{1}\right)$ is contained in a hyperplane in $\mathbb{R}^{n_{x}}$.

(iv). If $x_{0} \in \mathcal{X}\left(\mathcal{A}^{1}\right)$, then either $x_{0} \in \partial \mathcal{X}_{N}$, or $x_{0} \in \partial \mathcal{X}\left(\mathcal{A}^{2}\right)$ and $x_{0} \in \partial \mathcal{X}\left(\mathcal{A}^{3}\right)$ for some active sets $\mathcal{A}^{2}, \mathcal{A}^{3}$ that are non-degenerate at $k=0$.

(v). For each $x_{0} \in \mathcal{X}\left(\mathcal{A}^{1}\right), \mathbf{p}\left(x_{0}, \beta_{0}, \mathcal{A}^{1}\right)$ satisfies the optimality conditions of Theorem 6.1 for all $\beta_{0} \in\left[\beta_{0,-}, \beta_{0,+}\right]$, where $\mathbf{p}\left(x_{0}, \beta_{0,-}, \mathcal{A}^{1}\right)=\mathbf{p}\left(x_{0}, \mathcal{A}^{2}\right)$ and $\mathbf{p}\left(x_{0}, \beta_{0,+}, \mathcal{A}^{1}\right)=\mathbf{p}\left(x_{0}, \mathcal{A}^{3}\right)$.

Proof

Properties (i), (ii), (iv) and (v) follow from the complementarity conditions of Theorem 6.1 and the linearity and uniqueness of $\mathbf{p}$ as a function of $x_{0}$ and $\beta_{0}$ for given $\mathcal{A}$, whereas (iii) is a result of the compatibility condition (31), which applies to $x_{0}$ if $\mathcal{A}^{1}$ is degenerate at $k=0$, and of Assumption 7.1, which implies that $\beta_{0}$ is necessarily scalar.

Lemma 7.2 implies that, if $\mathcal{A}$ is degenerate at stage $k=0$, then $\mathbf{u}, \mathbf{w}$ are continuous in a neighbourhood of $x_{0} \in \mathcal{X}(\mathcal{A})$, whereas the multiplier sequences $\boldsymbol{\mu}, \boldsymbol{\nu}, \boldsymbol{\eta}$ are discontinuous at $x_{0} \in \mathcal{X}(\mathcal{A})$. As a result, although the optimal cost $J_{m}^{*}\left(x_{0}\right)$ is continuous in a neighbourhood of $x_{0} \in \mathcal{X}(\mathcal{A})$, the gradient of $J_{m}^{*}\left(x_{0}\right)$ is not uniquely defined. Due to the sequential min-max nature of (2), this causes non-uniqueness of the active set satisfying the optimality conditions of Theorem 6.1 at $x_{0} \in \mathcal{X}\left(\mathcal{A}^{1}\right)$, whenever $\mathcal{A}^{1}$ contains a degenerate subproblem at some stage $k>0$, i.e. there exists at least one other active set $\mathcal{A}^{2}$ such that $\mathcal{X}\left(\mathcal{A}^{1}\right) \subseteq \mathcal{X}\left(\mathcal{A}^{2}\right)$. This non-uniqueness of active sets implies that the globally optimal solutions of (2) can be discontinuous functions of the state if subproblems are degenerate at $k>0$. The active set algorithm described below avoids such discontinuities by considering active set changes that ensure the continuity of optimal control and disturbance sequences.

At each iteration $i=0,1, \ldots$ the solver determines $x_{0}^{(i+1)}$ and $\mathcal{A}^{(i+1)}$ such that $\mathcal{A}^{*}\left(x_{0}^{(i+1)}\right)=$ $\mathcal{A}^{(i+1)}$, where $\mathcal{A}^{(i+1)}$ is determined from $\mathcal{A}^{(i)}$ via a search over $x_{0} \in \mathcal{X}\left(\mathcal{A}^{(i)}\right)$ along the line $x_{0}=x_{0}^{(i)}+\alpha\left(x^{p}-x_{0}^{(i)}\right), \alpha \in \mathbb{R}$. Since $\mathcal{X}\left(\mathcal{A}^{(i)}\right)$ may overlap $\mathcal{X}\left(\mathcal{A}^{(i-1)}\right)$, the search direction is determined by the condition $x_{0} \in \mathcal{X}\left(\mathcal{A}^{(i)}\right)$. For the special case of a degenerate subproblem in $\mathcal{A}^{(i)}$ at stage $k=0$, the search is performed over $\beta_{0}$ with $x_{0}$ fixed. Each iterate, $x_{0}^{(i)}, i=0,1, \ldots$, satisfies the optimality conditions of Theorem 6.1 corresponding to $\mathcal{A}^{(i)}$, and furthermore the sequence $\left\{x_{0}^{(0)}, x_{0}^{(1)}, \ldots\right\}$ converges in a finite number of iterations to the plant state $x^{p}$.

\section{Algorithm 1}

Initialize with $x_{0}^{(0)}$ and an active set $\mathcal{A}^{(0)}$ such that $x_{0}^{(0)} \in \mathcal{X}\left(\mathcal{A}^{(0)}\right)$, and set $i:=0$. At iteration $i=0,1, \ldots$ :

1. Compute $\left\{P_{k}, q_{k}\right\}$ for $k=N-1, \ldots, 0$, and $\left\{\Phi_{k}, \phi_{k}\right\}$ for $k=0, \ldots, N-1$, and hence $\mathcal{X}\left(\mathcal{A}^{(i)}\right)$ using (34).

2. If $i=0$ :

$$
\alpha^{(i)}:=\max _{\alpha \leq 1}\left\{\alpha: x_{0}^{(i)}+\alpha\left(x^{p}-x_{0}^{(i)}\right) \in \mathcal{X}\left(\mathcal{A}^{(i)}\right)\right\} .
$$

Else if $\mathcal{A}^{(i)}$ is non-degenerate at $k=0$ :

$$
\alpha^{(i)}:=\max _{\alpha \leq 1}\left\{|\alpha|: x_{0}^{(i)}+\alpha\left(x^{p}-x_{0}^{(i)}\right) \in \mathcal{X}\left(\mathcal{A}^{(i)}\right)\right\} .
$$

Else:

$$
\beta_{0,-}^{(i)}:=\min _{\beta_{0} \in \mathbb{R}}\left\{\beta_{0}: \mathbf{p}\left(x_{0}^{(i)}, \beta_{0}, \mathcal{A}^{(i)}\right) \text { satisfies (34) }\right\}
$$


$\beta_{0,+}^{(i)}:=\max _{\beta_{0} \in \mathbb{R}}\left\{\beta_{0}: \mathbf{p}\left(x_{0}^{(i)}, \beta_{0}, \mathcal{A}^{(i)}\right)\right.$ satisfies (34) $\}$

and select $\beta_{0}^{(i)}$ corresponding to the active set that is different from the active set at the preceding iteration.

3. If $\mathcal{A}^{(i)}$ is degenerate at $k=0$ : set $x_{0}^{(i+1)}:=x_{0}^{(i)}, i:=i+1$ and update $\mathcal{A}^{(i)}$ using the new active constraints.

Else:

if $\alpha^{(i)}=1$, set $\mathcal{A}^{*}:=\mathcal{A}^{(i)}$, compute $u_{N}^{*}\left(x^{p}\right)$ and stop.

if $\alpha^{(i)}<1$, set $x_{0}^{(i+1)}:=x_{0}^{(i)}+\alpha^{(i)}\left(x^{p}-x_{0}^{(i)}\right), i:=i+1$, and update $\mathcal{A}^{(i)}$ using the new active constraints.

Return to step 1.

Remark 7.3

The origin, $x_{0}=0$, is assumed to be a feasible initial condition for Problem (2), therefore a trivial initialization for Algorithm 1 is the choice $x_{0}^{(0)}=0$ and $\mathcal{A}^{(0)}=\{\emptyset, \ldots, \emptyset\}$. In the context of MPC, further computational savings can be achieved by warm-starting Algorithm 1. This can be done by choosing $x_{0}^{(0)}$ at time $t+1$ equal to the plant state $x^{p}$ at time $t$. The active set can then be initialized in Algorithm 1 as $\mathcal{A}^{(0)}(t+1)=\mathcal{A}^{*}(t)$.

Theorem 7.4

For $x^{p} \in \mathcal{X}_{N}$, Algorithm 1 terminates after a finite number of iterations at $\mathcal{A}^{*}$ such that $\mathcal{A}^{*}=$ $\mathcal{A}^{*}\left(x^{p}\right)$.

Proof

Each iteration involves a line-search over one of the finite number of line segments that are defined by the intersections of the line containing $x^{(0)}$ and $x^{p}$ with the regions $\mathcal{X}(\mathcal{A})$ for all $\mathcal{A} \in \Omega$. The line-search is constructed so that $x_{0}^{(i+1)} \in \partial \mathcal{X}\left(\mathcal{A}^{(i)}\right) \cap \partial \mathcal{X}\left(\mathcal{A}^{(i+1)}\right)$ for all $i \geq 0$, and it follows from Lemma 7.2 that the active set change at each iteration is uniquely defined, and therefore the sequence $\left\{\mathcal{A}^{(i)}, i=0,1, \ldots\right\}$ is also uniquely defined and since the EP solution for each active set is uniquely defined in terms of $x_{0}$ and $\beta_{0}$, the sequence $\left\{\mathcal{A}^{(i)}, i=0,1, \ldots\right\}$ is therefore uniquely defined by $x_{0}^{(0)}$ and $x^{p}$. Furthermore, each active set can either appear at most once in the sequence $\left\{\mathcal{A}^{(i)}, i=0,1, \ldots\right\}$ or else the sequence is necessarily periodic. We show next that the cycling between active sets that is implied by the latter case cannot occur by considering separately the two methods described in Remark 7.3 for initializing the algorithm.

If Algorithm 1 is initialized with $x_{0}^{(0)}=0$, then the line segment joining $x_{0}^{(0)}$ and $x^{p}$ necessarily intersects the terminal set $\mathcal{X}^{f}$ and hence contains points at which Problem (2) is known to have a unique solution, namely the unconstrained optimal control law (since this satisfies the conditions of Theorem 6.1 within $\mathcal{X}^{f}$ by construction). It follows that $\mathcal{A}^{(0)}$ appears only once in the sequence $\left\{\mathcal{A}^{(i)}, i=0,1, \ldots\right\}$, and, because of the uniqueness of the active set change at each iteration, cycling is therefore not possible. Given that $x^{p} \in \mathcal{X}_{0}$ Lemma 7.2 implies that $\left\{\mathcal{A}^{(i)}, i=0,1, \ldots\right\}$ must in this case terminate at an active set $\mathcal{A}^{*}$ such that $x^{p} \in \mathcal{X}\left(\mathcal{A}^{*}\right)$. Finally this must occur after a finite number of iterations since the total number of possible active sets is finite.

Consider next the case that Algorithm 1 is initialized with arbitrary $x_{0}^{(0)} \in \mathcal{X}_{0}$ by warm-starting. There must exist a homotopy between the EP solutions in any two contiguous regions $\mathcal{X}(\mathcal{A})$ and $\mathcal{X}\left(\mathcal{A}^{\prime}\right)$ that intersect the line segment joining $x_{0}^{(0)}$ and $x^{p}$ since the preceeding argument (and the fact that $\mathcal{X}^{f}$ contains an open neighbourhood of the origin) implies that, starting from some point in $\mathcal{X}^{f}$, it is possible to construct by homotopy the EP solution along a line that intersects the boundary $\partial \mathcal{X}(\mathcal{A}) \cap \partial \mathcal{X}\left(\mathcal{A}^{\prime}\right)$ and to place this intersection point arbitrarily close to the intersection point of the line segment joining $x_{0}^{(0)}$ and $x^{p}$ with the same boundary. The existence of such a homotopy follows by a construction of successive partial homotopy paths which result from warm starting for subsequent time instants $t$. Therefore the sequence $\left\{\mathcal{A}^{(i)}, i=0,1, \ldots\right\}$ necessarily terminates at an active set $\mathcal{A}^{*}$ such that $x^{p} \in \mathcal{X}\left(\mathcal{A}^{*}\right)$, and this must be achieved in a finite number of iterations since $\Omega$ contains a finite number of active sets. 
The possibility of overlapping regions in $x_{0}$-space has the implication that the line-search path changes direction whenever a new degenerate subproblem is encountered.

Proposition 7.5

If, at iteration $i$ of Algorithm $1, \mathcal{A}^{(i)}$ is non-degenerate at $k=0$ and the number of linearly dependent constraints in $\mathcal{A}^{(i)}$ and $\mathcal{A}^{(i-1)}$ are different, then $\alpha^{(i)}$ and $\alpha^{(i-1)}$ are opposite in sign.

Proof

Consider first the case that, in the minimization at stage $k=1$, constraint $C 1$ is active and constraint $C 2$ is inactive at iteration $i-1$, while constraints $C 1$ and $C 2$ are both active at iteration $i$, where $C 1$ and $C 2$ are linearly dependent. The solution to this minimization subproblem therefore requires that a compatibility constraint $C 3$ is enforced at iteration $i$ in the maximization at stage $k=0$. Let $\alpha$ denote the line-search parameter during iteration $i$, and denote the multipliers of $C 2$ and $C 3$ as $\mu$ and $\zeta$ respectively. Then since $\mu=\zeta=0$ must satisfy the first order necessary conditions for $\alpha=0$, from (30) and (25) we obtain

$$
\begin{aligned}
& \mu=\mu^{\alpha} \alpha+\mu^{\beta} \beta \\
& \zeta=\zeta^{\alpha} \alpha-\beta
\end{aligned}
$$

for some constants $\mu^{\alpha}, \mu^{\beta}$ and $\zeta^{\alpha}$.

During the line-search at iteration $i$, Algorithm 1 sets $\zeta=0$, so that $\beta=\zeta^{\alpha} \alpha$, and $\mu=\left(\mu^{\alpha}+\right.$ $\left.\mu^{\beta} \zeta^{\alpha}\right) \alpha>0$. Consider instead choosing $\beta=-\mu^{\alpha} \alpha / \mu^{\beta}$ in order to make $C 2$ weakly active, and let $\alpha=\epsilon,|\epsilon| \ll 1$, i.e.

$$
\begin{aligned}
& \mu=0 \\
& \zeta=\left(\mu^{\alpha} / \mu^{\beta}+\zeta^{\alpha}\right) \epsilon .
\end{aligned}
$$

Since $\zeta$ is $O(\epsilon)$, removal of $C 3$ from the maximization problem at stage $k=0$ while $\alpha=\epsilon$ causes at most an $O(\epsilon)$ a change in any primal or dual variable. Hence the only constraint that can become active or inactive when $C 3$ is removed is $C 2$. Constraint $C 3$ acts on the maximization subproblem at $k=0$ so as to force $C 2$ to hold with equality in the minimization subproblem at $k=1$. But removing $C 3$ can only result in an increase in the maximization objective, and hence its removal has the same effect as tightening $C 2$ in the minimization subproblem. Thus, if we remove $C 3$, then $C 2$ becomes redundant, which implies that at this point in the line-search $x_{0}$ must lie in the active set region in which $C 1$ is active while $C 2$ and $C 3$ are inactive, namely $x_{0}^{(i)}+\epsilon\left(x^{p}-x_{0}^{(i)}\right) \in \mathcal{X}\left(\mathcal{A}^{(i-1)}\right)$. Therefore $\alpha^{(i)}$ and $\alpha^{(i-1)}$ must be of opposite sign.

By considering a reversal of the direction of the line-search, the same argument can be applied to the case in which the number of linearly dependent constraints is reduced by one. Furthermore there is no loss of generality in assuming that the linear dependent constraints occur in the minimization at stage $k=1$, since the same arguments also apply if $k>1$.

\section{Remark 7.6}

The formulation and properties of Algorithm 1 apply to subproblems with any number of linearly dependent constraints and also to degenerate subproblems occurring at more than one stage, which may lead to multiple overlapping regions $\mathcal{X}(\mathcal{A})$ at a given $x^{p}$. However Assumption 7.1 implies that at most two constraints can be linearly dependent at the initial stage; a greater number would correspond to the line-of-search intersecting an affine subspace of dimension $n_{x}-2$. If this (unlikely) situation occurs, it may be resolved by perturbation of the initial state $x^{p}$.

\section{Remark 7.7}

Overlapping regions $\mathcal{X}(\mathcal{A})$ do not occur either in the absence of disturbances (since the nominal problem formulation can be condensed to a single QP problem) or in the input-constrained problem formulation considered in [8,7], since this can have no linearly dependent constraints under Assumption 7.1. 
Remark 7.8

If $x^{p}$ is infeasible, Algorithm 1 necessarily terminates with an infeasible line-search over $\beta_{0}$ in step 2.

Remark 7.9

Provided that at a given $x^{p}$ the conditions of Theorem 4 are satisfied, then despite the possibility of overlapping regions along the line-of-search, the solution recovered at $x^{p}$ is the DP-optimal solution to Problem (2).

\subsection{Computation}

We next consider the online computational effort that is required to solve a single-stage degenerate subproblem in order to get a conservative estimate of the overall computational requirement. We make the assumption that (24) and (29) are solved using the null space method (see e.g. [11]). This approach, applied to (24), involves computing the QR decomposition of $\left[\begin{array}{cc}-D^{T} \hat{C}_{m}^{T} & G_{m}^{T}\end{array}\right]^{T}$, which requires $O\left(2\left(n_{G}+d_{u+}\right) n_{w}^{2}\right)$ floating point operations (where $n_{G}$ is the number of rows of $G_{m}$ and $d_{u+}$ is the order of degeneracy in the minimization at stage $m-1$ ); this can be further reduced using incremental rank-1 updates. Furthermore, the required matrix inversions and matrix multiplications require $O\left(a_{1} n_{w}^{3}+a_{2} n_{w}^{2}\left(n_{G}+d_{u+}\right)+a_{3} n_{w}\left(n_{G}+d_{u+}\right)^{2}\right)$ operations. Similarly, the solution of (29) requires $O\left(b_{1} n_{u}^{3}+b_{2} n_{u}^{2}\left(n^{f}+n_{E}+\hat{d}_{w}\right)+b_{3} n_{u}\left(n^{f}+n_{E}+\hat{d}_{w}\right)^{2}\right)$ operations (where $n_{G}$ and $n_{E}$ are the number of rows of $G_{m}, E_{m}$ and $\hat{d}_{w}$ is the order of degeneracy in the maximization at stage $m$ ). The constants $a_{1}, a_{2}, a_{3}$ and $b_{1}, b_{2}, b_{3}$ depend on the implementation of the null space method and the underlying functions used for the Cholesky and QR decompositions. The other significant contribution comes from the matrix multiplications in (28) and (32b), which require $O\left(6 n_{x}^{3}+2 n_{x}^{2}\left(n_{w}+n_{w}+n_{E}+\hat{d}_{w}+d_{u}\right)+2 n_{x}\left[\hat{d}_{w} d_{u}+\left(n_{E}+\hat{d}_{w}\right)\left(n_{u}+d_{u+}\right)\right]\right)$ operations. Combining the above estimates for $N$ stages gives an $O(N)$ dependence of computational complexity. Noting that the computation required for the forward simulation is $O\left(n_{x}^{2} N\right)$ (since only the projection, $\Phi_{t}\left(x^{p}-x_{0}^{(i)}\right)$, of $\Phi_{t}$ in $(38 \mathrm{a}, \mathrm{b})$ is needed), and also that the computation involved in the line search in step (ii) is comparatively insignificant, we estimate the computation per iteration of Algorithm 1 to grow as $O(N)$.

Thus the dependence of computation per iteration on the horizon length $N$ is linear. The required number of iterations is problem-dependent and depends on whether exact representations of the robust controllability sets are used (as in Section 9). Furthermore, the number of iterations can be minimized using warm-starts (as described in Remark 7.3). The computational load is in stark contrast to existing schemes for min-max receding horizon control, which, for the case of optimal approaches that are based on dynamic programming, have computational loads that depend exponentially on $N$ (see e.g. [24, 15]). Likewise, the approach of [12] based on a suboptimal (affine) controller parameterization requires the solution of a semidefinite program (SDP) in a number of optimization variables that grows quadratically with the horizon length and, as illustrated in Section 9, this typically results in a much higher computational load than Algorithm 1.

\section{CLOSED LOOP STABILITY AND $l_{2}$-GAIN BOUND}

This section defines a receding horizon control law based on Algorithm 1 which ensures that: (i) Problem (2) is recursively feasible (i.e. if a solution exists at a given plant state $x_{t}$, then a solution necessarily exists at $x_{t+1}$, for any $t \geq 0^{\ddagger}$ ); (ii) $\mathcal{X}_{N}$ is robustly invariant; (iii) $\gamma$ is an upper bound on the $l_{2}$-gain from $w_{t}$ to $\left(Q^{1 / 2} x_{t}, R^{1 / 2} u_{t}\right)$ if $\sum_{t=0}^{\infty}\left\|w_{t}\right\|^{2}$ is infinite; (iv) $x=0$ is asymptotically stable with region of attraction $\mathcal{X}_{N}$ if $\sum_{t=0}^{\infty}\left\|w_{t}\right\|^{2}$ is finite. If Algorithm 1 recovers the globally optimal solution of Problem (2) at all times, then the receding horizon control law has a fixed horizon length $N$ and standard stability results apply [6]. However, to account for the possibility that the

${ }_{\ddagger}^{\ddagger \text { In this section } x_{t}}, u_{t}, w_{t}$ denote the state, control and disturbance input of system (1) at time $t$. 
solution of (2) is only locally optimal (e.g. because of the presence of degenerate subproblems), the horizon length is reduced whenever a specified condition on the optimal value of the min-max cost at successive time instants is violated.

\section{Definition 8.1}

A set $\mathcal{S} \subseteq \mathbb{R}^{n_{x}}$ is robust controlled positively invariant (RCPI) for (1) under the control law $u=\kappa(x)$ if: (i) $\mathcal{S} \subseteq\{x \in \mathcal{X}: \kappa(x) \in \mathcal{U}\}$; and (ii) $A x+B \kappa(x)+D w \in \mathcal{S}$ for all $x \in \mathcal{S}$ and all $w \in \mathcal{W}$.

It is assumed that the terminal set $\mathcal{X}^{f}$ is an RCPI set for (1) under the optimal, unconstrained, infinite horizon control law $u=u^{f}(x)$. We also assume $\mathcal{X}^{f} \subseteq\left\{x \in \mathbb{R}^{n_{x}}: w^{f}\left(x, u^{f}(x)\right) \in \mathcal{W}\right\}$, so that the unconstrained optimal (worst-case) disturbance law $w^{f}\left(x, u^{f}(x)\right)$ satisfies the disturbance bounds at every point in $\mathcal{X}^{f}$.

The receding horizon control law at time $t$ is defined by the solution $u_{N_{t}}^{*}\left(x_{t}\right)$ of Problem (2) with horizon length $N_{t}$, which is chosen as follows.

Algorithm 2

At $t=0$ set $N_{0}:=N$. For all $t>0$, if

$$
\frac{1}{2}\left(\left\|x_{t-1}\right\|_{Q}^{2}+\left\|u_{N_{t-1}}^{*}\left(x_{t-1}\right)\right\|_{R}^{2}-\gamma^{2}\left\|w_{t-1}\right\|^{2}\right)+J_{N_{t-1}}^{*}\left(x_{t}\right)>J_{N_{t-1}}^{*}\left(x_{t-1}\right),
$$

then set $N_{t}:=N_{t-1}-1$; otherwise set $N_{t}:=N_{t-1}$.

Note that it is possible to check at time $t$ whether the condition in (41) is satisfied since $w_{t-1}$ can be determined time $t$ given knowledge of $x_{t}$.

\section{Theorem 8.2}

If $x_{0} \in \mathcal{X}_{N}$, then for any admissible disturbance sequence $\left\{w_{t} \in \mathcal{W}, t=0,1, \ldots\right\}$, the state of (1) with $u_{t}=u_{N_{t}}^{*}\left(x_{t}\right)$ satisfies $x_{t} \in \mathcal{X}_{N}$ for all $t \geq 0$. Furthermore, if $N_{t}=N$ for all $t$, then

$$
\sum_{t=0}^{\infty}\left(\left\|x_{t}\right\|_{Q}^{2}+\left\|u_{t}\right\|_{R}^{2}\right) \leq \gamma^{2} \sum_{t=0}^{\infty}\left\|w_{t}\right\|^{2}+2 J_{N}^{*}\left(x_{0}\right),
$$

otherwise the following bound holds for some finite $\Delta$,

$$
\sum_{t=0}^{\infty}\left(\left\|x_{t}\right\|_{Q}^{2}+\left\|u_{t}\right\|_{R}^{2}\right) \leq \gamma^{2} \sum_{t=0}^{\infty}\left\|w_{t}\right\|^{2}+2 J_{N}^{*}\left(x_{0}\right)+\Delta
$$

Proof

The recursive feasibility of Problem (2) and robust invariance of $\mathcal{X}_{N}$ under the control law of Algorithm 2 follow from the constraint $x_{t+1} \in \mathcal{X}_{N_{t}-1}$ in (2a) and the nested property $\mathcal{X}_{N} \supseteq \cdots \supseteq$ $\mathcal{X}_{1} \supseteq \mathcal{X}^{f}$, which is a consequence of the definition of $\mathcal{X}_{m}$ in (2e) and the RCPI property of $\mathcal{X}^{f}$.

To prove the bound in (42), we first demonstrate a monotonicity property of the optimal value of the cost. By optimality we have $J_{m-1}^{*}(x)=J_{m-1}\left(x, u_{m-1}^{*}(x)\right)$ and $J_{m}^{*}(x) \leq J_{m}\left(x, u_{m-1}^{*}(x)\right)$ since $u_{m-1}^{*}(x)$ is feasible but suboptimal for the $m$-stage minimization subproblem for $x \in \mathcal{X}_{m-1}$, implying

$$
J_{m}^{*}(x)-J_{m-1}^{*}(x) \leq \hat{J}_{m}^{*}\left(A x+B u_{m-1}^{*}(x)\right)-\hat{J}_{m-1}^{*}\left(A x+B u_{m-1}^{*}(x)\right) .
$$

Similarly, for the maximization subproblem we obtain $\hat{J}_{m-1}^{*}(\hat{x}) \geq \hat{J}_{m-1}\left(\hat{x}, w_{m}^{*}(\hat{x})\right)$ and $\hat{J}_{m}^{*}(\hat{x})=$ $\hat{J}_{m}\left(\hat{x}, w_{m}^{*}(\hat{x})\right)$, so that

$$
\begin{aligned}
\hat{J}_{m}^{*}(\hat{x})-\hat{J}_{m-1}^{*}(\hat{x}) & \leq \hat{J}_{m}\left(\hat{x}, w_{m}^{*}(\hat{x})\right)-\hat{J}_{m-1}\left(\hat{x}, w_{m}^{*}(\hat{x})\right) \\
& =J_{m-1}^{*}\left(\hat{x}+D w_{m}^{*}(\hat{x})\right)-J_{m-2}^{*}\left(\hat{x}+D w_{m}^{*}(\hat{x})\right)
\end{aligned}
$$

Since $J_{0}$ satisfies the generalized Riccati equation by assumption, it follows for the 1-stage problem that

$$
\left.J_{1}^{*}(x)-J_{0}^{*}(x)=\min _{u \in \mathcal{U}} \max _{w \in \mathcal{W}}\left\{\frac{1}{2}\|x\|_{Q}^{2}+\frac{1}{2}\|u\|_{R}^{2}-\frac{1}{2} \gamma^{2}\|w\|^{2}\right)+J_{0}^{*}(A x+B u+D w)-J_{0}^{*}(x)\right\} \leq 0
$$


and by induction we therefore have

$$
J_{m}^{*}(x)-J_{m-1}^{*}(x) \leq 0 \quad \forall m=1, \ldots, N,
$$

which is the monotonicity property of the optimal cost.

Next consider the case that Algorithm 1 recovers the globally optimal solution of Problem (2) at all times $t \geq 0$ and apply the monotonicity property (44) together with optimality of the cost for the $N_{t}$-stage problem. The optimal cost is given by

$$
J_{N_{t}}^{*}(x)=\min _{u \in \mathcal{U}(x)} \max _{w \in \mathcal{W}}\left\{\frac{1}{2}\left(\|x\|_{Q}^{2}+\|u\|_{R}^{2}-\gamma^{2}\|w\|^{2}\right)+J_{N_{t}-1}^{*}(A x+B u+D w)\right\}
$$

and by optimality of the maximization we have

$$
\frac{1}{2}\left(\|x\|_{Q}^{2}+\left\|u_{N_{t}}^{*}(x)\right\|_{R}^{2}-\gamma^{2}\left\|w_{t}\right\|^{2}\right)+J_{N_{t}-1}^{*}\left(A x+B u_{N_{t}}^{*}(x)+D w_{t}\right) \leq J_{N_{t}}^{*}(x) \quad \forall w_{t} \in \mathcal{W}
$$

Applying the monotonicity property (44) and setting $x=x_{t}$ yields

$$
\frac{1}{2}\left(\left\|x_{t}\right\|_{Q}^{2}+\left\|u_{N_{t}}^{*}\left(x_{t}\right)\right\|_{R}^{2}-\gamma^{2}\left\|w_{t}\right\|^{2}\right)+J_{N_{t}}^{*}\left(x_{t+1}\right) \leq J_{N_{t}}^{*}\left(x_{t}\right) .
$$

Therefore (41) cannot hold, so $N_{t}=N$ for all $t$, and the $l_{2}$-gain bound (42) follows by summing both sides of this inequality for $t=0,1, \ldots$.

However, if $u_{t}=u_{N_{t}}^{*}\left(x_{t}\right)$ is feasible but suboptimal for Problem (2) with $N_{t}$ stages, then the monotonicity property (44) does not necessarily hold. In this case we introduce an additional term $\delta_{t}\left(w_{t}, x_{t}\right)$ to account for the increase in the value of the predicted cost when condition (41) holds:

$$
\begin{aligned}
\frac{1}{2}\left(\left\|x_{t}\right\|_{Q}^{2}+\left\|u_{N_{t}}^{*}\left(x_{t}\right)\right\|_{R}^{2}-\gamma^{2}\left\|w_{t}\right\|^{2}\right) & +J_{N_{t}}^{*}\left(A x_{t}+B u_{N_{t}}^{*}\left(x_{t}\right)+D w_{t}\right) \\
& \leq J_{N_{t}}^{*}\left(x_{t}\right)+\delta_{t}\left(w_{t}, x_{t}\right) \leq J_{N_{t}}^{*}\left(x_{t}\right)+\tilde{\delta}_{t} \quad \forall w_{t} \in \mathcal{W}
\end{aligned}
$$

where $\tilde{\delta}_{t}$ is an upper bound on $\delta_{t}\left(w_{t}, x_{t}\right)$, which is obtained by maximizing over all admissible $x_{t}$ and $w_{t}$ for the given horizon $N_{t}$. Furthermore $J_{N_{t}}^{*}\left(x_{t}\right)$ is necessarily finite for all $t$, and the suboptimality gaps $\tilde{\delta}_{t}$ are incurred at a finite number of instances due to Algorithm 2. This is because the horizon can shrink at most $N$ times, after which $x_{t}$ will remain in $\mathcal{X}^{f}$ for all $t$. Hence if (41) holds at time instants $t_{1}, \ldots, t_{N}$, then we necessarily obtain, for finite $\Delta_{1}=2 \sum_{i=1}^{N} \tilde{\delta}_{t_{i}}$ and $\Delta_{2}=2 \sum_{i=1}^{N} J_{N_{t_{i}+1}}^{*}\left(x_{t_{i}+1}\right)-J_{N_{t_{i}}}^{*}\left(x_{t_{i}+1}\right)$ :

$$
\sum_{t=0}^{\infty}\left(\left\|x_{t}\right\|_{Q}^{2}+\left\|u_{N_{t}}^{*}\left(x_{t}\right)\right\|_{R}^{2}\right) \leq \gamma^{2} \sum_{t=0}^{\infty}\left(\left\|w_{t}\right\|^{2}\right)+2 J_{N}^{*}\left(x_{0}\right)+\Delta_{1}+\Delta_{2}
$$

implying the bound (43) with $\Delta=\Delta_{1}+\Delta_{2}$.

Corollary 8.3

If $\sum_{t=0}^{\infty}\left\|w_{t}\right\|^{2}<\infty$, then $x=0$ is asymptotically stable with region of attraction equal to $\mathcal{X}_{N}$. If $\sum_{t=0}^{\infty}\left\|w_{t}\right\|^{2}$ is infinite, then $x=0$ is stable and the following $l_{2}$-gain bound holds:

$$
\lim _{T \rightarrow \infty} \frac{1}{T} \sum_{t=0}^{T}\left(\left\|x_{t}\right\|_{Q}^{2}+\left\|u_{t}\right\|_{R}^{2}\right) \leq \gamma^{2} \lim _{T \rightarrow \infty} \frac{1}{T} \sum_{t=0}^{T}\left\|w_{t}\right\|^{2} .
$$

Proof

Stability of $x=0$ follows from optimality of $u^{f}(x)$ in the absence of constraints and the RCPI property of $\mathcal{X}^{f}$. If $\sum_{t=0}^{\infty}\left\|w_{t}\right\|^{2}$ is finite, then (42) and (43) imply $\lim _{t \rightarrow \infty} x_{t}=0$ and thus asymptotic stability. Otherwise (42) and (43) imply the bound (45).

\section{Remark 8.4}

In practice $\gamma$ should be chosen large enough such that the concave condition (35a) holds for all active sets likely to be encountered. If (35a) does not hold at some stage, then our procedure involves a shrinking of the horizon to ensure the conditions of Theorem 8.2 as described in Algorithm 2. An interesting avenue for future research would be to consider mechanisms for systematically increasing $\gamma$ online so that (35a) is satisfied and for subsequently restarting Algorithm 1. 


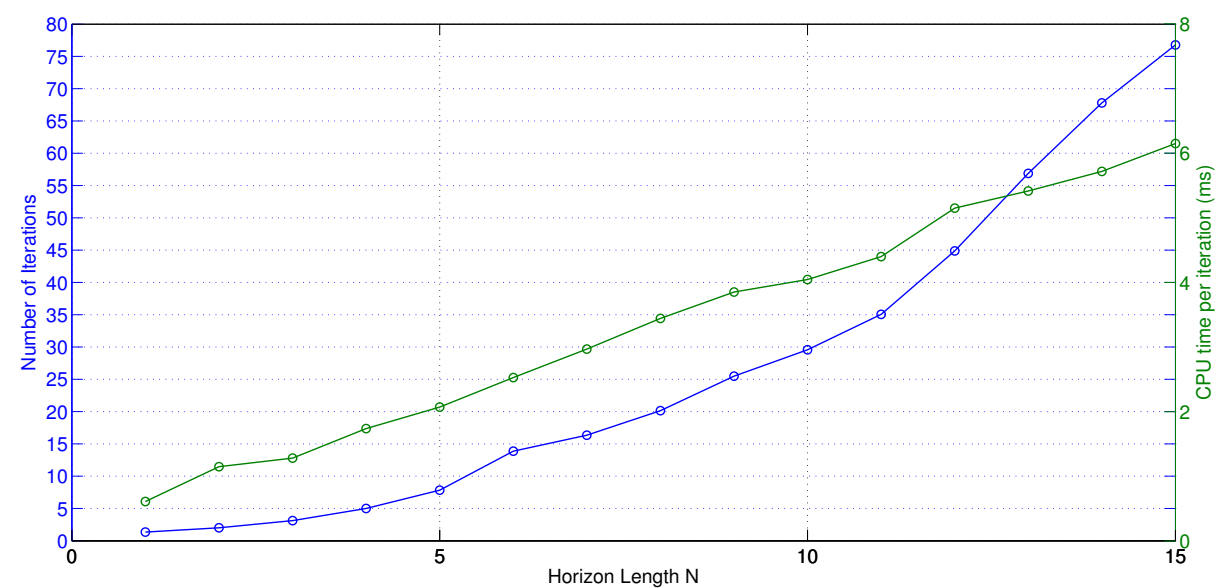

Figure 1. Number of iterations and time per iteration vs horizon length using a $2.4 \mathrm{GHz}$ i5 processor

\section{NUMERICAL EXAMPLES}

\subsection{Example 1}

To demonstrate the performance of the robust active set algorithm consider the simplified linear model of the pitch dynamics of an aircraft as in [25]:

$$
\begin{aligned}
J \ddot{\alpha}+b \dot{\alpha} & =C_{Z E} l E-\left(C_{Z E} l+C_{C W} d\right) \alpha \\
m \ddot{h} & =\left(C_{Z E}+C_{C W}\right) \alpha-C_{Z E} E
\end{aligned}
$$

where $m$ and $J$ denote the mass and moment or inertia about the pitch axis, $C_{Z E}$ and $C_{C W}$ are the elevator lift and wing lift coefficients respectively and $b$ is a friction coefficient. Here $d, l$ are the distances between center of gravity and center of lift and between center of gravity and the point where the elevator lift force is applied. We denote the state by $x=[\alpha, \dot{\alpha}, h, \dot{h}]^{T}$, where $\alpha, h$ denote the pitch angle (in degrees) and vertical height $(\mathrm{m})$ of the aircraft. The input is the elevator angle, i.e. $u=E$. We further assume that there is uncertainty in the form of additive disturbances in the aerodynamic forces and torques acting on the aircraft. We take the parameters as in [25] to be $J=1, m=1, b=4, C_{Z E}=1, C_{Z W}=5, l=3, d=0.2$. The resulting continuous time model is discretized with a sampling time of $T=0.2 \mathrm{~s}$, giving model parameters in (1) as

$$
A=\left[\begin{array}{rrrc}
0.938 & 0.134 & 0 & 0 \\
-0.536 & 0.402 & 0 & 0 \\
0.119 & 0.007 & 1 & 0.2 \\
1.174 & 0.092 & 0 & 1
\end{array}\right] \quad D=\left[\begin{array}{ll}
0 & 0 \\
1 & 0 \\
0 & 0 \\
0 & 1
\end{array}\right] \quad B=\left[\begin{array}{r}
0.046 \\
0.402 \\
-0.019 \\
-0.180
\end{array}\right]
$$

The constraint sets are given by $\mathcal{U}=\{u \in \mathbb{R}:-25 \leq u \leq 25\}$ and $\mathcal{W}=\left\{w \in \mathbb{R}^{2}:-0.25 \leq w_{i} \leq\right.$ 0.25 for $i=1,2\}$, and cost weights by $Q=\operatorname{diag}[0,0,0.04,0], R=2.5 \times 10^{-4}$. By solving the generalized Riccati equation, $P, u^{f}$, and $w^{f}$ were computed with $\gamma$ chosen sufficiently large to satisfy conditions (35a,b) of Theorem 6.1. For $N=15$ this gives $\gamma^{2}=5$ and $u^{f}(x)=K_{u}^{f} x$, $w^{f}(x, u)=K_{w}^{f} \hat{x}$,

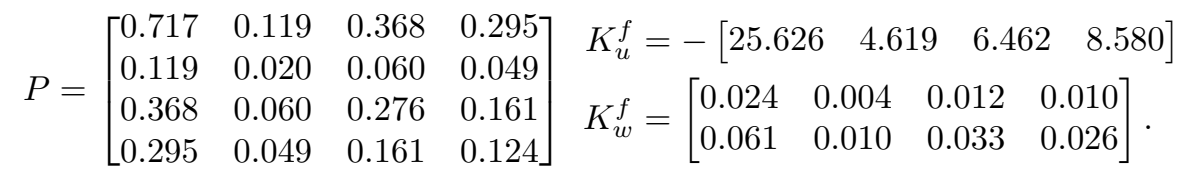

A robust positive invariant set $\mathcal{X}^{f}$ was obtained using the procedure of [16] and the robust controllability sets $\mathcal{X}_{m}$ for $m=1, \ldots, 15$ were computed offline as in ([4]) and are enforced online 
as explicit state constraints using Algorithm 1. To illustrate how the number of iterations and time per iteration varies with horizon length, 400 plant states were sampled inside $\mathcal{X}_{N} \backslash \mathcal{X}_{N-1}$ for $N=1, \ldots, 15$. The time per iteration grows linearly with horizon length in agreement with the theoretical results (see Figure 1). The growth of the number of iterations with horizon length depends on the example chosen, but can be explained by the increasing complexity of the robust controllability sets $\left(\mathcal{X}_{15}\right.$, for example, is described by 231 inequalities). For $N=15$ the average time per iteration is $6.24 \mathrm{~ms}$ and the average number of iterations 77.31 (total time $0.48 \mathrm{~s}$ ). The numerical examples were performed in Matlab using a $2.4 \mathrm{GHz}$ i5 processor.
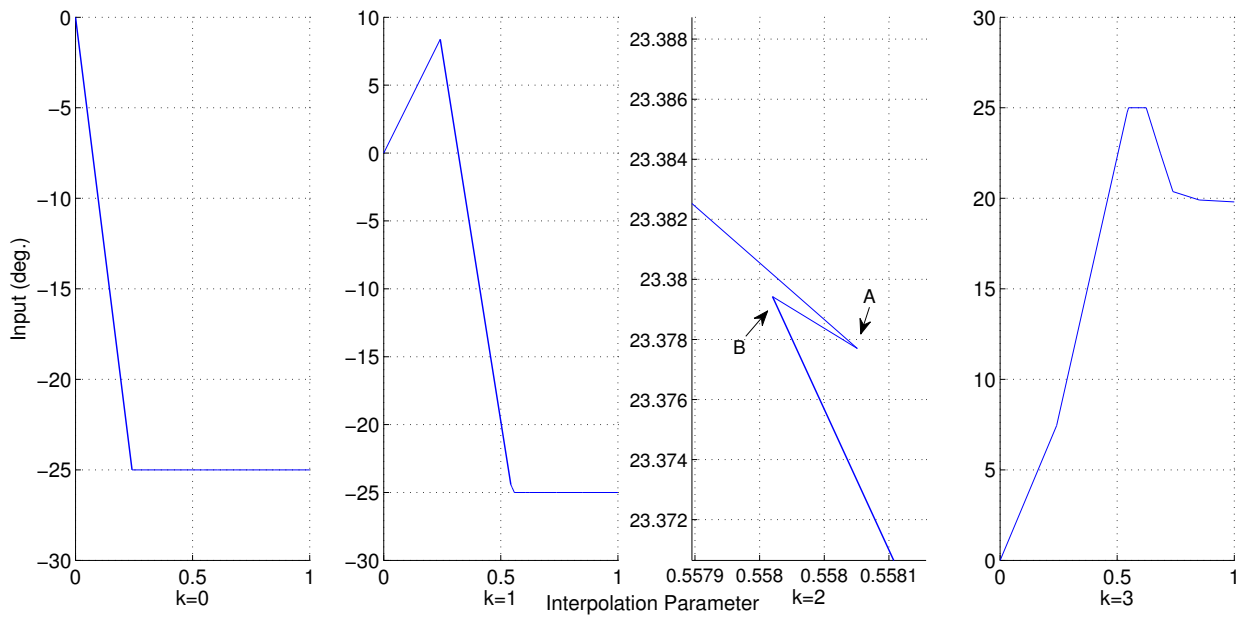

Figure 2. Input $u=E$ as function of interpolation parameter $(\mathrm{N}=4)$
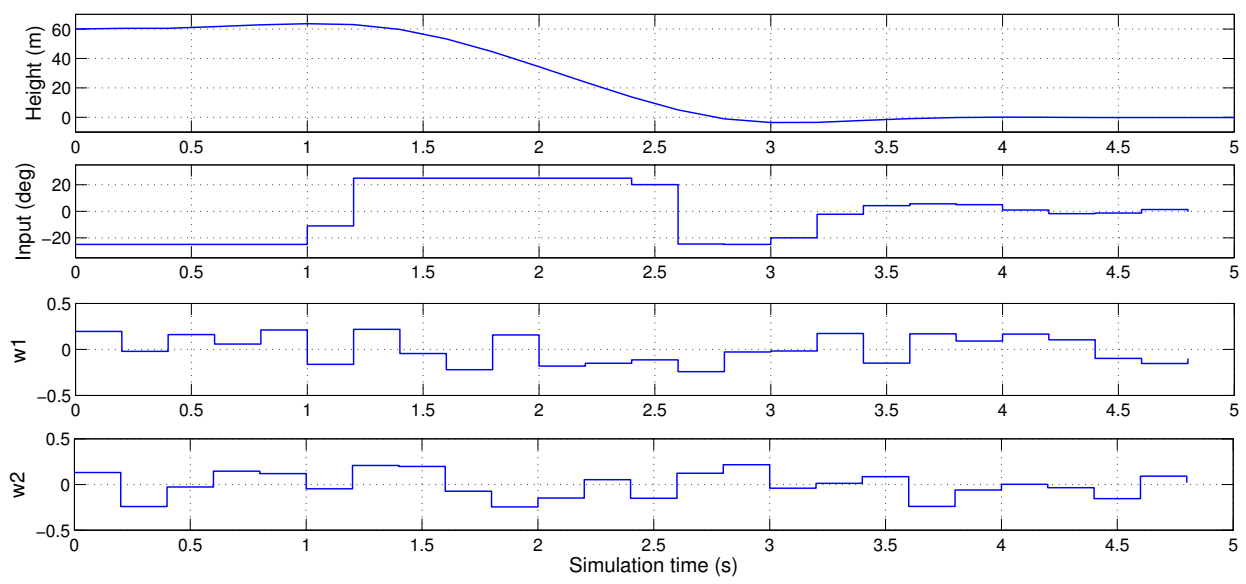

Figure 3. Closed Loop Response of Aircraft height $\left(x_{3}\right)$ for $N=10$

Next we use the non-minimum phase dynamics of this example to illustrate the effects of degenerate input and state constraints. For $N=4$ the input profiles are depicted as functions of the interpolation parameter (which lies in $[0,1]$ and the algorithm is cold started) in Figure 2. The control law is a piecewise affine function of this parameter and overlapping regions are possible due to non-unique Lagrange multipliers. This situation occurs for example in the control input for stage $k=2$ shown in Fig. 2. Here a forward step (inside a region corresponding to non-degenerate constraints) to point $\mathrm{A}$ is followed by a backward step (in which constraints are degenerate) to point $B$, then another forward step (again with non-degenerate constraints). The required changes in direction of the line search are implemented simply by invoking conditions (34a), (34b), (36a), (36b) at the region boundaries. The active set algorithm avoids the discontinuous solutions that 


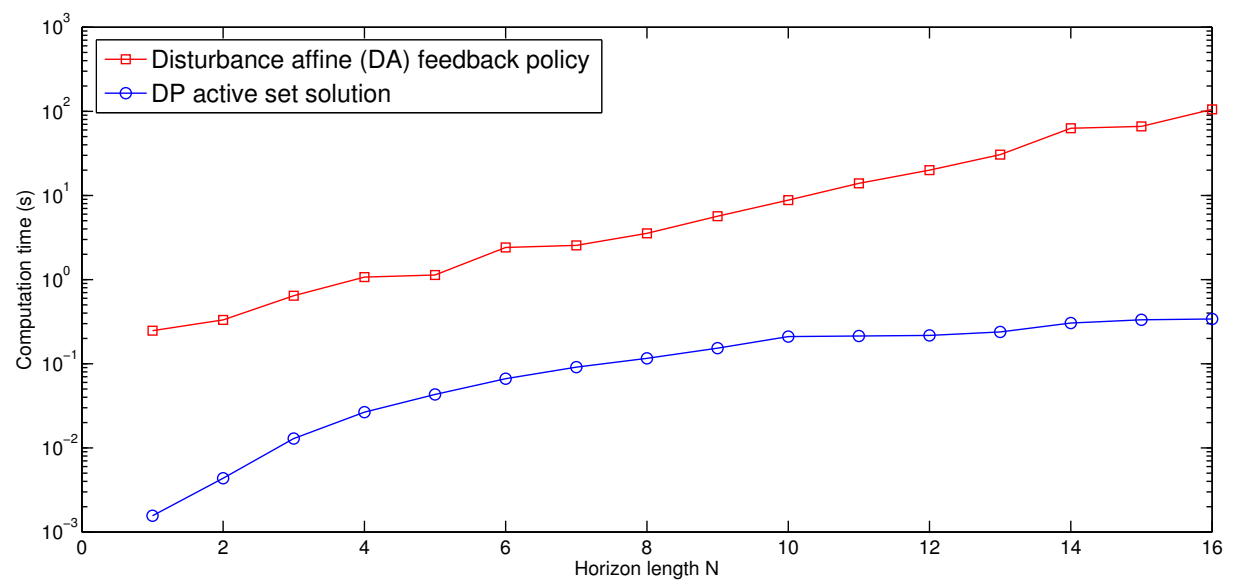

Figure 4. Execution times of DP active set algorithm and Disturbance Affine Policy for Example 2 using a $2.3 \mathrm{GHz}$ i 7 processor.

could occur if the line-search always moved in the forward direction towards the current plant state by considering active set changes that ensure the continuity of optimal solution trajectories. The closed loop response under the receding horizon controller is shown in Figure 3, where aircraft height $x_{3}$ is the controlled output.

\subsection{Example 2}

Algorithm 1 was used to compute an optimal control law for the uncertain controlled triple integrator defined by (1) with

$$
A=\left[\begin{array}{lll}
1 & 1 & 0 \\
0 & 1 & 1 \\
0 & 0 & 1
\end{array}\right], \quad B=\left[\begin{array}{l}
0 \\
0 \\
1
\end{array}\right], \quad D=\left[\begin{array}{lll}
1 & 0 & 0 \\
0 & 1 & 0 \\
0 & 0 & 1
\end{array}\right]
$$

and with state constraint set $\mathcal{X}=\left\{x \in \mathbb{R}^{3}:-500 \leq x_{1} \leq 5\right\}$, input constraint set $\mathcal{U}=\{u \in$ $\mathbb{R}:|u| \leq 4\}$, disturbance set $\mathcal{W}=\left\{w \in \mathbb{R}^{3}:\left|w_{i}\right| \leq 0.25, i=1,2,3\right\}$, and cost weights $Q=$ $\left[\begin{array}{lll}1 & 0 & 0\end{array}\right]^{T}\left[\begin{array}{lll}1 & 0 & 0\end{array}\right], R=0.1$.

$P, K_{u}^{f}$, and $K_{w}^{f}$ were computed for $\gamma^{2}=50$, giving

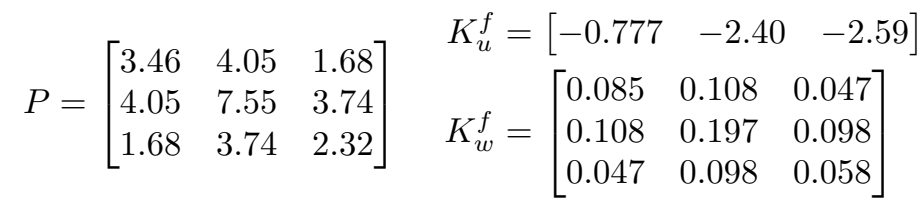

Fig. 4 compares the computational load of the exact active set algorithm of Alg.1 (implemented in Matlab) with that required by a Disturbance Affine (DA) feedback policy [19] applied to Problem (2). The DA policy was formulated as a semidefinite program (as in [12]), and execution times were obtained using SeDuMi [26]. The results were obtained for a set of 50 plant states $x$ sampled inside the set $\mathcal{X}_{N}^{D A} \backslash \mathcal{X}_{N-1}^{D A}$, for $N=1, \ldots, 16$ and in each case Algorithm 1 was initialized with $x_{0}^{(0)}=0$. This illustrates that the active set algorithm is at least an order of magnitude faster than the DA approach. The feasible sets for the DA policy and for DP are showin in Fig. 5 and indicate a significant increase in size when using a DP approach. The predicted performance for these plant states using the DA policy was found to be around $4 \%$ suboptimal (where in order to provide a meaningful comparison plant states were selected for which no non-concave subproblems occured). 

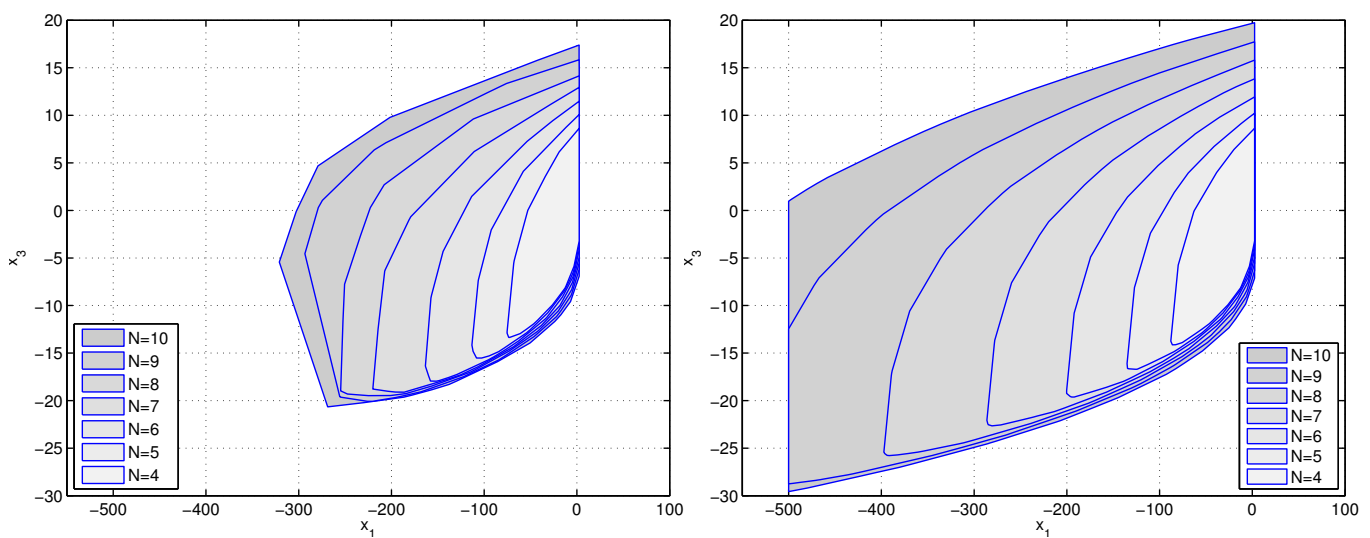

Figure 5. Feasible sets for DA-Policy (left) and DP-Policy (right) for Example 2, projected onto the $x_{1}-x_{3}$ subspace

\section{CONCLUSIONS}

This paper proposes an active set algorithm for the robust min-max MPC problem for input- and state-constrained linear systems with bounded disturbances. The relevent optimality conditions are derived and an active set strategy based on homotopy is formulated which applies both to degenerate and non-degenerate subproblems. The effects of the presence of degenerate subproblems on the optimality of the solution and on the convergence of the proposed algorithm are discussed.

Numerical results confirm significant improvements both in terms of computational efficiency and performance over the robust receding horizon control strategy based on disturbance-affine parameterizations. An interesting future research direction is the application of homotopy-based active set methods to min-max robust MPC for linear systems with polytopic model uncertainty and bounded disturbances. Another promising extension of the method would consider the feasibility problem associated with Problem (2) using a sequential min-max dynamic programing problem with a piecewise linear stage cost, which could therefore be tackled using multiparametric linear programming. In contrast to the global solution to the feasibility problem assumed in Problem (2), the feasibility problem would then be solved locally online for the current plant state.

\section{REFERENCES}

1. A. Bemporad, F. BorRelli, AND M. MoRARI, Min-max control of constrained uncertain discrete-time linear systems, IEEE Trans. Automatic Control, 48(9) (2003), pp. 1600-1606.

2. D. BERTSEKAS AND I. RHODES, On the minimax reachability of target sets and target tubes, Automatica, 7 (1971), pp. 233-247.

3. M. BEST, An algorithm for the solution of the parametric quadratic programming problem, in Applied Mathematics and Parallel Computing, Physica-Verlag: Heidelberg, 1996, pp. 57-76.

4. F. Blanchini And S. Miani, Set-Theoretic Methods in Control, Birkhauser, 2008.

5. A. BRYSON AND Y.-C. Ho, Applied optimal control, Hemisphere, 1975.

6. J. BUERGER, M. CANNON, AND B. KOUVARITAKIS, An active set solver for min-max robust control, in American Control Conference, 2013, pp. 4227-4233.

7. $\frac{}{161}$, An active set solver for input-constrained robust receding horizon control, Automatica, 1 (2014), pp. 155161.

8. M. Cannon, W. Liao, and B. Kouvaritakis, Efficient MPC optimization using Pontryagin's minimum principle, Int. J. Robust Nonlinear Control, 18 (8) (2008), pp. 831-844.

9. M. DIEHL AND J. BJÖRNBERG, Robust dynamic programming for min-max model predictive control of constrained uncertain systems, IEEE Trans. Automatic Control, 49 (2004), pp. 2253-2257.

10. H. FERREAU, H. BOCK, AND M. DIEHL, An online active set strategy to overcome the limitations of explicit MPC, Int. J. Robust Nonlinear Control, 18 (2008), pp. 816-830.

11. R. FLETCHER, Practical Methods of Optimization, Wiley, 2000.

12. P. Goulart, E. KerRigan, AND T. Alamo, Control of constrained discrete-time systems with bounded $l_{2}$ gain, IEEE Trans. Automatic Control, 54 (2009), pp. 1105-1111. 
13. P. Goulart, E. KerRigan, AND J. MaCiEJOWSKI, Optimization over state feedback policies for robust control with constraints, Automatica, 42 (2006), pp. 523-533.

14. C. JONES, P. GRIEDER, AND S. RAKOVIC, A logarithmic-time solution to the point location problem for parametric linear programming, Automatica, 42 (2006), pp. 2215-2218.

15. E. KeRRIGAN AND J. MACIEJOWSKI, Robustly stable feedback min-max model predictive control, in Proc. ACC, Denver, CO, 2003.

16. I. KOLMANOVSKY AND E. GILBERT, Theory and computation of disturbance invariant sets for discrete-time linear systems, Mathematical Problems in Engineering, 4 (1998), pp. 317-367.

17. M. Kothare, V. BALAKrishnan, AND M. MORARI, Robust constrained model predictive control using linear matrix inequalities, Automatica, 32 (1996), pp. 1361-1379.

18. J. LEE AND Z. YU, Worst-case formulations of model predictive control for systems with bounded parameters, Automatica, 33 (1997), pp. 763-781.

19. J. LÖFBERG, Minimax approaches to robust model predictive control, $\mathrm{PhD}$ thesis, Linköping University, Sweden, 2003.

20. D. Mayne, S. Raković, R. Vinter, AND E. KerRigan, Characterization of the solution to a constrained $H_{\infty}$ optimal control problem, Automatica, 42 (2006), pp. 371-382.

21. D. Mayne, J. Rawlings, C. Rao, And P. Scokaert, Constrained model predictive control: Stability and optimality, Automatica, 36 (2000), pp. 789-814.

22. D. MAYNe, M. SERON, AND S. RAKOviĆ, Robust model predictive control of constrained linear systems with bounded disturbances, Automatica, 41 (2005), pp. 219-224.

23. S. Raković, B. Kouvaritakis, M. Cannon, C. Panos, And R. Findeisen, Parameterized tube model predictive control, IEEE Trans. Automatic Control, 57 (2012), pp. 2746-2761.

24. P. SCOKAERT AND D. MAYNE, Min-max feedback model predictive control for constrained linear systems, IEEE Trans. Automatic Control, 43 (1998), pp. 1136-1142.

25. J. Slotine AND W. LI, Applied Nonlinear Control, Prentice-Hall, 1991.

26. J. STURM, Using SeDuMi 1.02, a Matlab toolbox for optimization over symmetric cones, Optimization Methods and Software, 11-12 (1999), pp. 625-653.

27. H. WitsenhaUSEn, A minimax control problem for sampled linear systems, IEEE Transactions on Automatic Control, 13 (1968), pp. 5-21. 